\title{
Gesta Romanorum
}

Der gemeinsame Nenner der Kapitel, die den zweiten Teil der vorliegenden Untersuchung bilden, liegt darin, dass sie von einzelnen fiktiven Kompositionen Leverkühns und darauf aufbauenden intermedialen Transpositionen bzw. Bezugnahmen handeln. Damit in den drei Kapiteln dieses Teils Leverkühns kompositorische Entwicklungen kohärent und nachvollziehbar dargestellt werden können, werden die drei fiktiven Werke, nämlich die Gesta Romanorum, die Apocalipsis cum figuris und die Dr. Fausti Weheklag, der Chronologie der intradiegetischen Zeitbezüge des Romans entsprechend behandelt: Zeitblom liefert seiner Leser*innenschaft präzise Daten zur Komposition und Aufführung der Kompositionen.

Das vorliegende Kapitel beschäftigt sich mit Leverkühns Puppenspiel Gesta Romanorum und zwei intermedialen Transpositionen des Werkes, jeweils von Beyer (Die Gesta Romanorum - Musik des Adrian Leverkühn, 1990) und Odegard (The Calling of St. Gregory, 1988). In den Ausführungen der ersten, dem Roman gewidmeten Abschnitte des Kapitels kristallisiert sich der Labor-Charakter der Gesta-Kapitel aus Thomas Manns Doktor Faustus heraus, die einerseits auf der intradiegetischen Ebene der Narration einer Experimentierphase im Schaffen Adrian Leverkühns entsprechen, andererseits eine Transitionsphase in Thomas Manns Arbeit am Motiv des Inzests in einem mythischen Kontext darstellen. In diesem Prozess, der anhand der drei Werke Wälsungenblut, Doktor Faustus und Der Erwählte kurz geschildert wird, ergeben sich aus intramedialer Sicht sowohl Kontinuitäten als auch Differenzen, etwa der durchgehende Rückgriff auf die Parodie und das In-Frage-Stellen der mythischen Vorlage samt Hervorhebung der Gefährlichkeit von Mythen sowie die - trotz Einbettung in unterschiedliche geographische Räume und Bezug auf zwei unterschiedliche Religionen - enge Verknüpfung von Inzest und Rassenfrage. 
Der zweite Teil des Kapitels widmet sich zwei intermedialen Transpositionen von Leverkühns Gesta romanorum. Beyers Die Gesta romanorum - Musik des Adrian Leverkühn befindet sich in einem Spannungsverhältnis zwischen Rekonstruktion und Adaption der Vorlage: Einerseits orientiert sich Besetzung und Stil an Thomas Manns Roman, anderseits wird das Ziel einer größeren Zugänglichkeit der Musik nicht durch das Medium des Puppenspiels, sondern durch das des Fernsehens verfolgt. In Odegards The Calling of St. Gregory wird das Motiv des Inzests jenseits von kulturellen Tabus behandelt; die spätmittelalterliche Exempelsammlung wird in Frage gestellt, indem gleichzeitig der parodistische Charakter von Leverkühns fiktiver Vertonung im plurimedialen Medium der Medienkombination verstärkt wird. Beide intermediale Produkte konzentrieren sich auf das zweite von Leverkühns vertonte Exempel, nämlich auf Gregors Mythos.

\subsection{Die Gesta Romanorum als Labor: ${ }^{1}$ Adrian Leverkühns Puppenspiel}

Der vorigen Darstellung des Inhalts des Kapitels entsprechend wird hier zunächst einmal auf Leverkühns fiktives Puppenspiel Gesta Romanorum eingegangen. Das Werk Gesta Romanorum ist eine anonyme spätmittelalterliche Exempelsammlung: Die sehr verschiedenen Texte samt ihren diversen Handlungen, Figuren und Stoffen haben hauptsächlich eines gemeinsam, nämlich ihre moralisch-didaktische Absicht. Die Exempelsammlung hat viele Schriftsteller inspiriert, etwa Shakespeare, Boccaccio, Lessing und Hofmannsthal, denn Werke dieser Autoren stützen sich auf einige Legenden aus der Sammlung. ${ }^{2}$ Auch im Schaffen Thomas Manns

\footnotetext{
${ }^{1}$ Der Begriff wurde dem später erwähnten Aufsatz Braidottis entnommen: Es entspricht der deutschen Übersetzung des von ihr verwendeten Wortes ,laboratory“. Zur Übersetzung: Braidotti: Politik der Affirmation. Übers. v. Elisa Barth. Leipzig: Merve 2018, S. 79.

${ }^{2}$ Vgl. Wawrznyiak, Udo: Gesta Romanorum. In: Brednich u. a. (Hrsg.): Enzyklopädie des Märchens. Handwörterbuch zur historischen und vergleichenden Erzählforschung, Bd. 5 von 14. Berlin/New York: de Gruyter 1987, S. 1201-1212. Nach der Auffassung von Weigand und Murdoch bedient sich Thomas Mann der Gesta-Übersetzung von Gräße, auf die sich das vorliegende Kapitel ebenfalls beziehe. Vgl. Murdoch, Brian: Gregorius. An Incestuous Saint in Medieval Europe and Beyond. Oxford: Oxford University Press 2012, S. 200; Weigand, Hermann J.: Thomas Mann's Gregorius. In: Germanic Review 27 (1952) H. 0031, S. 10-30, hier: S. 10; Gesta Romanorum. Übers. v. Johann G. T. Gräße. Leipzig: Löffler 1905 [Dresden 1842], 3. Aufl. Bd. 1. Siehe auch: Weiske, Brigitte: Gesta Romanorum (2 Bände: 1. Untersuchungen zu Konzeption und Überlieferung, 2. Texte, Verzeichnisse). Tübingen: Niemeyer 1992.
} 
stellen die Gesta den Hypotext ${ }^{3}$ zweier Werke dar, und zwar nicht nur des hier behandelten Romans, Doktor Faustus, sondern auch eines zweiten, später entstandenen, nämlich Der Erwählte. Während Doktor Faustus auf zwei Exempel ${ }^{4}$ des Hypotextes Bezug nimmt, die jeweils die Motive des Ehebruchs und des Inzests in den Vordergrund rücken und auf der intradiegetischen, histoire-spezifischen Ebene in der ersten Phase des Ersten Weltkriegs (1914-15) von Adrian Leverkühn vertont werden, basiert der 1951 erschienene Roman lediglich auf dem Exempel des Papstes Gregor. ${ }^{5}$ Im intramedialen Prozess wird daher der Fokus auf den mythischen Hypotext reduziert, gleichzeitig aber die Geschichte des christlichen Ödipus Gregor an sich ausführlicher behandelt und - nach den Worten des Autors - zum Hauptstoff seines „kleinen archaischen Roman[s]“ (Ent: 114).

In Bezug auf Adrian Leverkühns vertonte Texte aus den Gesta taucht sowohl im Roman als auch in den ersten Beiträgen aus der Forschungsliteratur - nicht selten das Wort ,Regression' auf. ${ }^{6}$ Zeitblom ist beispielsweise der Auffassung, dass „die ,Gesta ' tatsächlich etwas wie eine ,Regression“ auf den musikalischen Stil von „Love's Labour Lost““ (DF: 465), ein Werk der frühen Phase Leverkühns, darstellen und äußert sogar seine Unzufriedenheit mit dem Werk und den damaligen Überlegungen seines Freundes über ,eine Kunst ohne Leiden, seelisch gesund, unfeierlich, untraurig-zutraulich, eine Kunst mit der Menschheit auf du und du“ (DF: 469). In einem 1952 publizierten Aufsatz von Weigand findet sich diese Stellungnahme ebenfalls: „The style of Adrian Leverkühn's puppet-play music was a reversion to the simpler manner of his earlier period“". ${ }^{7}$ Erst in späteren Beiträgen zu Thomas Manns Roman unterliegt diese Auffassung einer Revision: Herrmanns betont beispielsweise, dass diese Schaffensperiode in Leverkühns Leben doch nicht völlig ,unschöpferisch[]“8 gewesen sei. Das vorliegende Kapitel versucht, jenseits der starren Dichotomien unschöpferisch/schöpferisch bzw. Regression/Progression zu argumentieren. Diese scheinen nicht angemessen, dem dynamischen Labor-Charakter der Gesta-Kapitel - sowohl auf der intradiegetischen Dimension des Romans als auch im Schaffen Thomas Manns - Rechnung zu tragen, der im Zentrum der folgenden Ausführungen steht.

\footnotetext{
${ }^{3}$ Hier und im weiteren Verlauf der Studie aus: Genette: Palimpsestes, z. B. S. 11.

${ }^{4}$ Thomas Mann sieht zunächst fünf Geschichten aus den Gesta vor, benutzt dann nur zwei. Siehe Hermanns, Ulrike: Thomas Manns Roman Doktor Faustus im Lichte von Quellen und Kontexten. Frankfurt am Main: Lang 1994, S. 40.

${ }^{5}$ Siehe Mann, Thomas: Der Erwählte. Frankfurt am Main: Fischer 2015 [1951], 30. Aufl.

${ }^{6}$ Zur Forschungsliteratur vgl. z. B. den bereits erwähnten Aufsatz von Weigand (Thomas Mann's Gregorius, Fußnote 2).

${ }^{7}$ Weigand: Thomas Mann's Gregorius, S. 11.

${ }^{8}$ Hermanns: Thomas Manns Roman, S. 38.
} 
Die Kapitel profilieren sich in Anlehnung an eine Publikation von Rosi Braidotti als vieldimensionales ,laboratory for the new in the sense of the actualizations of experiments in becoming ". 9 Im Rahmen dieses Experimentierens kommt dem Motiv des Inzests eine große Bedeutung zu: Auch die Gesta setzen sich mit der Frage: „[W]hat happened to the heirs of Oedipus?"10 auseinander, die u. a. auf den Diskurs des Begehrens, genauer genommen auf den eines „desire outside cultural intelligibility“ ${ }^{11}$ verweist.

In der bereits erwähnten Schrift beschreibt Braidotti, was sie unter einem ,intensiven Text ${ }^{\star}$ versteht: ${ }^{12}$

The literary text as an experiment in sustainable models of change is a laboratory grounded in accurate knowledge and subjected to the same rigorous rules of verification as science or philosophy. This fundamental parallelism cuts across different areas, disciplines and textual genres. Life, science and art are equally enlisted to the project of experimenting with transformations.

Auch Leverkühns fiktives Experimentieren entspringt genauen und sorgfältigen Vorarbeiten und Lektüren, die Zeitblom wie folgt schildert:

Auf dem Tische lagen ein paar Bücher: ein Bändchen Kleist, worin das Lesezeichen bei dem Aufsatz über die Marionetten eingelegt war, ferner die unvermeidlichen Sonette Shakespeares und noch ein Band mit Stücken dieses Dichters, - „Was ihr wollt“ war darin, ,Viel Lärm um nichts“, und, wenn ich nicht irre, auch „Die beiden Veroneser". (DF: 444 f.)

Durch diese Lektüren setzt sich Leverkühn u. a. mit Marionettentheater und Narzissmus ${ }^{13}$ (Kleist), Geschwisterinzest (Shakespeares Was ihr wollt) und ehelicher Treue (Shakespeares Viel Lärm um Nichts) auseinander; darüber hinaus liest Leverkühn ausschließlich Komödien von Shakespeare: Ein Hinweis darauf,

\footnotetext{
${ }^{9}$ Braidotti: Intensive Genre, S. 48.

${ }^{10}$ Butler, Judith: Antigone's Claim. Kinship Between Life and Death. New York: Columbia University Press 2000, S. 25.

${ }^{11}$ Ebd., S. 54.

${ }^{12}$ Braidotti: Intensive Genre, S. 48.

${ }^{13}$ Wie Svenja Frank hervorhebt, deutet Kleists Text „,bekanntlich die Bewegungen eines Jünglings als Inbegriff der Anmut, die dieser, nachdem er sich unvermittelt im Spiegel erblickt, nicht mehr wiederholen kann: Seine Anmut bleibt nach dem Erwachen der Selbst-Wahrnehmung für immer verloren“. Frank, Svenja: Inzest und Autor-Imago im Marionettentheater. Zum Identitätskonzept in Felicitas Hoppes Paradiese, Übersee. In: Holdenried, Michaela (Hrsg., in Zusammenarbeit mit Stefan Hermes): Felicitas Hoppe: Das Werk. Berlin: Schmidt 2015, S. 49-68, hier: S. 65.
} 
dass er für die mittelalterliche Exempelsammlung keine Realisierung in aristotelischer tragischer Handlung vorsieht. Leverkühn entscheidet sich für die Form des Marionettentheaters und vertieft seine Kenntnisse durch Besuche von Puppenbauern und -spielen sowie das Studium der ,sehr kunstreichen Handpuppen- und Schattenfiguren-Spiele der Javanen“ (DF: 466). Er nimmt also sowohl in seinen Vorarbeiten an den Gesta als auch in der Kompositionsphase selbst ,keine Rücksicht auf die Trennungen verschiedener Felder, Disziplinen und Textgattungen“. 14 Wo dieser Aspekt im Schaffen Leverkühns verstärkt wird, ist in der Apocalipsis cum figuris. Dort kommt es zu einer „Vermischung der Stimmen, Gattungen und Codes“ ${ }^{15}$ wie Zeitblom seiner Leser*innenschaft mitteilt:

Am Horizont, ich bin dessen sicher, stand schon damals, wahrscheinlich schon seit Ausbruch des Krieges, der ja für eine Divination, wie die seine, einen tiefen $\mathrm{Ab}$ - und Einschnitt, die Eröffnung einer neuen, tumultuösen und grundstürzenden, mit wilden Abenteuern und Leiden überfüllten Geschichtsperiode bedeutete, - am Horizont seines schöpferischen Lebens stand bereits die „Apocalipsis cum figuris“, das Werk, das diesem Leben einen schwindelnden Auftrieb geben sollte, und bis zu welchem - so sehe wenigstens ich den Prozeß - er sich mit den genialischen Puppen-Grotesken die Wartezeit vertrieb. (DF: $458 \mathrm{f}$.)

Zeitblom schreibt der Gesta zwar Genialität zu, definiert sie jedoch zugleich als Zeitvertrieb und - wie eingangs dieses Unterabschnitts bereits angesprochen als Regression, was einen seiner vielen Widersprüchen in seinem Erzählen darstellt. Hierbei muss allerdings angemerkt werden, dass er ebenfalls eine gewisse Prozesshaftigkeit erkennt, von der er auch in der Lage ist, ein Ziel zu sehen, und zwar die Apocalipsis cum figuris. Nicht nur gelten die Gesta als Vorbereitung auf das apokalyptische Werk, vor allem in Anbetracht der Aufhebung von Trennungen etwa zwischen Stilen, Gattungen und Disziplinen einerseits, ihrer intensiven Vermischung andererseits sowie der Auseinandersetzung mit dem Kulturgut aus dem Mittelalter. Sie antizipieren und bereiten auch auf das letzte Werk, die $D r$. Fausti Weheklag, vor, wie im Folgenden aufgezeigt wird. Kleists Text Über das

\footnotetext{
${ }^{14}$ Braidotti: Politik der Affirmation, S. 48. Erwähnenswert ist hier auch, dass Leverkühn „lockere Szenarien und ungefähre Wechselreden entwarf, worauf [...] [Zeitblom] es war, der sie in Mußestunden rasch in ihre endgültige, aus Prosa und Reimverschen gemischte Form brachte“, DF: 461.

${ }^{15}$ Derrida, Jacques: Apokalypse. Hrsg. v. Peter Engelmann, übers. v. Michael Wetzel. Wien: Böhlau 1985 [1983], S. 76.
} 
Marionettentheater, den Leverkühn als Vorbereitung auf die Gesta liest, konzentriert sich bekanntlich auf die Anmut des Mechanischen, das immerhin mit „Empfindung betrieben werden“16 kann.

Das Studium des Marionettentheaters sowie die Lektüre von Kleists Text stellen eine wichtige Etappe im kompositorischen Werdegang Leverkühns dar, da sie ihm eine zum selben Zeitpunkt der histoire stattfindende Reflexion über die Möglichkeit eines ,Durchbruch $[\mathrm{s}][\ldots]$ aus geistiger Kälte in eine Wagniswelt neuen Gefühls“ (DF: 468; Herv. i. O.) erlauben. Dieser Durchbruch gelingt Leverkühn im Roman durch seine letzte Komposition, die Dr. Fausti Weheklag, welche die einzige ist, die „seine Idee eines ,strengen Satzes “ entwickelt[]“ (DF: 704). ${ }^{17}$ Die Weheklag bestätigt die Auffassung des Tänzers in Kleists Text, der mit der Erzählinstanz spricht, von einer möglichen „Rekonstruktion des Ausdrucks“ (DF: 703) auch durch das Mechanische, wofür in Doktor Faustus die Zwölftontechnik stehen könnte, die zum ersten Mal in Leverkühns letzter Komposition angewendet wird. ${ }^{18}$ Nicht zuletzt betrachtet Kleists Text Über das Marionettentheater das Puppenspiel als effiziente technische Vorbereitung: Ein Tänzer, der sich ausbilden möchte, könne viel von der Pantomimik der Puppen lernen. Auch Leverkühn bereitet sich, allerdings als Komponist und nicht als Tänzer, durch die Form des Puppenspiels auf seine späteren zwei Kompositionen, die Apocalipsis und die Weheklag, vor.

In diesem Prozess des „,becoming“19 spricht Leverkühn laut Zeitbloms Wiedergabe der Ereignisse auch darüber, wie er zum künstlerischen Durchbruch gelangen möchte. Leverkühn beklagt, die Musik habe zu seiner Zeit ,im Dienst technischer Geistigkeit“ (DF: 468) an „Gefühlswärme“ (ebd.) verloren und wünscht sich ,eine Wiedergewinnung des Vitalen und der Gefühlskraft" (ebd.). Er phantasiert von einer Kunst, die, um dem Absterben entgegenzuwirken, „den Weg zum ,Volk““ (DF: 469) wiederfinden könnte und bedient sich dementsprechend eines „volksnahen Instruments““20 nämlich des Puppenspiels. Betrachtet man aber, wie die

\footnotetext{
${ }^{16}$ Der Aufsatz erschien in den Berliner Abendblättern, 63.- 66. Blatt, den 12.-15. Dezember 1810. Hier aus: Kleist, Heinrich von: Über das Marionettentheater. In: Sembdner, Helmut (Hrsg.): Kleists Aufsatz über das Marionettentheater. Studien und Interpretationen. Berlin: Schmidt 1967, S. 9-16, hier: S. 10.

${ }^{17}$ Zeitblom assoziiert diesen Durchbruch ebenfalls auf der intradiegetischen Dimension der Narration politisch mit dem Bestreben Deutschlands, durch den Ersten Weltkrieg ,zur dominierenden Weltmacht“ (DF: 402) zu werden.

${ }^{18}$ Vgl. auch Malknecht, Ludovica: Un'etica di suoni. Musica, morale e metafisica in Thomas Mann. Mailand: Mimesis 2010, S. 177 f. Vgl. auch Kap. 5.

${ }^{19}$ Braidotti: Intensive Genre, S. 49.

${ }^{20}$ Hermanns: Thomas Manns Roman, S. 38.
} 
Mehrheit seiner Stücke im Roman durch zahlreiche intermediale Systemerwähnungen beschrieben werden, so scheint das Ziel einer größeren Zugänglichkeit der Musik keine Leitidee seines Schaffens zu sein. Zwar mischt Leverkühn etwa in der Apocalipsis Jazz und französischen Impressionismus, jedoch selten aus der Absicht heraus, ,,eine Kunst mit der Menschheit auf du und du“ (DF: 469) zu produzieren, um ein breiteres Publikum für seine Kompositionen zu gewinnen. Dies wird besonders deutlich, wenn man die intermedialen Einzelreferenzen auf Werke und Stile von Komponist*innen der Neuen Musik in die Analyse einbezieht: Um einige Beispiele aus dem nächsten Kapitel zu nennen, seien die Denaturierung des Klanges und die Umwertung von Konsonanz und Dissonanz in der Apocalipsis, die auf kompositorische Experimente u. a. der Wiener Schule um Arnold Schönberg hinweisen, erwähnt.

Wenn Leverkühns Absicht ab den Gesta romanorum wirklich darin bestünde, ein größeres Publikum zu erreichen, dann müsste man feststellen, dass dieser Leitgedanke in Zeitbloms Schilderungen seiner Werke nicht mehr als tragend erscheint. Plausibler scheint, dass dieser Widerspruch in Leverkühns Kompositionswerdegang der Unzuverlässigkeit des eifersüchtigen Erzählers zuzuschreiben ist, der anlässlich dieser Konversationen das weitere Diskutieren mit Leverkühn unterlässt und „vielmehr ein besorgtes Auge auf Rudi Schwerdtfeger [hat], ob der ihn am Ende nicht wieder umarmen wollte“ (DF: 470). ${ }^{21}$ Zugleich gilt es hervorzuheben, dass der Prozess des Werdens, der in dieser kompositorischen Phase Leverkühns besonders evident scheint, durchaus auch von „,block of becoming[s]",22 also von Blöcken, Brüchen und Zögerungen charakterisiert ist. Folglich werden laut Braidotti im Laufe dieses Prozesses einige Potenziale aktualisiert, andere beiseitegelassen. In Bezug auf Leverkühns kompositorische Phase des Puppenspiels Gesta romanorum könnte resümierend gesagt werden, dass etwa die Absicht einer größeren Ausdruckskraft der musikalischen Sprache weiterverfolgt wird, das Ziel aber einer größeren Zugänglichkeit seiner Werke und seiner musikalischen Sprache jedoch nicht.

Adrian Leverkühn bereitet sich auf das Puppenspiel-Werk auch durch die Lektüre von Shakespeares Komödien vor, auch weil Zeitblom zufolge „die ,Gesta“ in ihrer historischen Unbelehrtheit, christfrommen Didaktik und moralischer Naivität [...] [i]m höchsten Grade [...] danach [sic] angetan [waren], Adrians parodistischen Sinn aufzuregen“ (DF: 459 f.). Leverkühns fiktive Medienkombination

\footnotetext{
${ }^{21}$ Die Erzählinstanz Serenus Zeitblom spricht Leverkühns Musik in den meisten Fällen die Kommunikationsfähigkeit ab, um bei anderer Gelegenheit hervorzuheben, wie elitär und erfolglos die Musik seines Freundes (besonders in Deutschland) sei. Siehe etwa DF: 446. ${ }^{22}$ Braidotti: Intensive Genre, S. 49.
} 
bietet daher eine Interpretation der Gesta an, die vom moralisch-didaktischen Zweck der Vorlage ,auf eine recht destruktive Weise“ (DF: 466) Abstand nimmt, indem „das Skurrile, besonders auch im Erotischen Possenhafte, an die Stelle moralischer Priesterlichkeit trat, aller inflationärer Pomp der Mittel abgeworfen und die Aktion der an sich schon burlesken Gliederpuppen-Bühne übertragen wurde“ (ebd.). Nach Zeitbloms Worten wird also in Leverkühns Puppenspiel Priesterlichkeit ins Skurrile und Erotische umgewertet und auf eine Theaterform übertragen, die sich für den ,pparodistischen Sinn“ Leverkühns besser eignet als die Gattung der Exempelsammlung. Der Erzähler liefert seiner Leser*innenschaft Hinweise darauf, wie Leverkühn die Vorlage liest und dementsprechend in seine Medienkombination transferiert und transformiert: Es handelt sich um kein rekonstruktives, sondern um ein adaptives Textverständnis, das einerseits durch Leverkühns Lesart bedingt und andererseits vom gewählten Medium des Puppenspiels abhängig ist. Zudem zeichnet sich hier Leverkühn als ,practitioner of deconstruction“ 23 aus, der ,within the terms of the system“24 arbeitet, „but in order to breach it““ ${ }^{25}$ Das System, in dem Leverkühn arbeitet, ist das der moralischen, mittelalterlichen Exempelsammlungen mythischen Stoffs, das die Dichotomie moral/immoral ins Zentrum stellt und diese durch christliche Argumentationen begründet. Der Ehebruch z. B., von dem die erste vertonte Geschichte handelt, lässt sich aus christlicher Perspektive sowohl anhand der Zehn Gebote als auch der Todsünden (luxuria) für immoral erklären. Durch Leverkühns Medienkombination wird die Vorlage nicht wirklich adaptiv, sondern eher „destruktiv“ gelesen: Das Werk scheint auf keine „Schlichtung von Gegensätzen“26 abzuzielen, was laut Börnchen die logische Ableitung der verwendeten Auffassung von Komik und Humor ist. Vielmehr werden Gegensätze etwa durch den nach Zeitblom skurrilen Charakter des Werkes sichtbar gemacht und ausgehalten. ${ }^{27}$ Um mit Derrida zu argumentieren, könnte man dementsprechend über Leverkühns Puppenspiel Folgendes sagen: Die „spielerische Bewegung innerhalb dieses Abstands zwischen den beiden Zeichen“",28 in diesem Fall: Moralität und

${ }^{23}$ Culler, Jonathan: On Deconstruction. Theory and Criticism after Structuralism. London (u. a.): Routledge \& Kegan 1983 [Cornell 1982], S. 86.

${ }^{24}$ Ebd.

${ }^{25}$ Ebd.

${ }^{26}$ Börnchen: Kryptenhall, S. 83.

${ }^{27}$ Siehe ebd.

${ }^{28}$ Derrida, Jacques: Positionen. Gespräch mit Jean-Louis Houdebine und Guy Scarpetta (1968). In: Ders.: Positionen. Gespräche mit Henri Ronse, Julia Kristeva, Jean-Louis Houdebine, Guy Scarpetta. Wien: Passagen 2009 [Paris 1972], 2., überarb. Aufl., S. 63-122, S. 94. 
Immoralität, bewirkt eine „umstürzende Dekonstruktion“, 29 die einer Deplatzierung in ein neues Medium ,außerhalb der Gegensätze“30 bedarf. In diesem Fall ermöglicht folglich die profane und auf die Unterhaltung abzielende Form des Puppenspiels eine Aufhebung der Trennung zwischen Moralität und Immoralität. Der Umwertung von Himmel und Hölle bzw. von Konsonanz und Dissonanz in der Apocalipsis liegt daher ein interpretatorisches Verfahren zugrunde, mit dem sich der fiktive Komponist von Doktor Faustus bereits auskennt und mit dem er in der Vertonung der Gesta ebenfalls experimentiert. Auch die Parodie eignet sich zur Umsetzung dieses Vorhabens und stellt eine Kontinuität in Leverkühns Schaffen dar, an der er im Labor der Gesta weiterarbeitet.

Nun zum ersten von Leverkühn vertonten Text aus den Gesta: Der Text trägt den Titel „Von der gottlosen List der alten Weiber“31 (DF: 460) und handelt von einem jungen Mann, der ,eine edle und sogar ausnehmend ehrbare Ehefrau“ (ebd.) begehrt, ,deren vertrauensvoller Gatte sich auf Reisen befindet“ (ebd.) und die sich von einer „Hexe“ (ebd.) durch eine List überzeugen lässt, mit dem jungen Mann zu schlafen, um nicht Gefahr zu laufen, in eine Hündin verwandelt zu werden. Das erste wichtige Motiv, das in der Gesta-Vertonung von Leverkühn zum Tragen kommt, ist das des Ehebruchs; diesbezüglich ergibt sich eine Interaktion zwischen Meta- und Intradiegese, denn das Motiv des Ehebruchs taucht später wieder auf und es lassen sich inhaltliche Entsprechungen zwischen dem hier beschriebenen Ehebruch und dem von Ines Institoris und Rudolf Schwerdtfeger feststellen. ${ }^{32}$ Die Darstellung des Ehebruchs beruht in der Literatur nicht selten auf „,medialen Interventionen“:33 Schwerdtfeger ist anwesend, als Leverkühn die Partitur der Gesta vorspielt. Zugleich gilt es hervorzuheben, dass Ines Institoris an diesen abendlichen Treffen im Nike-Saal nicht teilnimmt. Das vermittelte Begehren ${ }^{34}$ durch das Medium der Musik könnte sich infolgedessen

\footnotetext{
${ }^{29}$ Ebd.

${ }^{30} \mathrm{Ebd}$.

${ }^{31}$ Der Titel wird wortwörtlich der Vorlage entnommen. Siehe Gesta Romanorum: S. 49-52.

${ }^{32}$ Diesbezüglich ist zu bemerken, dass hier Zeitblom auch die Rolle des metadiegetischen Erzählers übernimmt. Dazu vgl. auch Elsaghe, Yahya: Das Goldene Horn und die Hörner der Männchen: Zur Krise der Männlichkeit in Doktor Faustus und Mario und der Zauberer. In: Honold, Alexander u. Niels Werber (Hrsg.): Deconstructing Thomas Mann. Heidelberg: Winter 2012, S. 121-134, hier: S. 125.

${ }^{33}$ Scherpe, Klaus: Der Buchstabe „A“ und andere Medien des Ehebruchs im Roman. Goethe, Hawthorne, Flaubert, Fontane, Tolstoi und Thomas Mann. In: Weimarer Beiträge 56 (2010) H. 3, S. 389-403, hier: S. 389, Herv. i. O.

${ }^{34}$ Siehe Girard, René: Deceit, Desire, and The Novel. Self and Other in Literary Structure. Übers. v. Yvonne Freccero. Baltimore: John Hopkins 1965 (insb. S. 26-37).
} 
eher auf das zwischen Leverkühn und Schwerdtfeger beziehen - wie auch auf das zwischen Leverkühn und Schildknapp, der ihm die Gesta vorliest, ${ }^{35}$ also durch das Medium der Literatur. Der oft eifersüchtige Zeitblom assoziiert dieses Begehren zwar mit keinem Ehebruch im eigentlichen Sinne, aber immerhin mit einem Verrat an seiner Liebe zu seinem Freund: Es wundert nicht, dass er der Leser*innenschaft Schwerdtfegers emotionale Reaktion auf Leverkühns Vorspiel detailliert beschreibt. ${ }^{36}$

Diese knappe Darstellung des ersten Textes leitet zum zweiten Schritt der Analyse über, der sich, ausgehend vom zweiten vertonten Exempel zu Gregorius, mit dem Inzest-Motiv im Schaffen Thomas Manns befasst.

\subsection{Doktor Faustus als Labor: Das Motiv des Inzests im Werk Thomas Manns}

Es gilt nun, die Kapitel über die Gesta im Gesamtwerk Thomas Manns zu kontextualisieren und sich dabei den daraus resultierenden intramedialen Bezügen zu widmen, die den Roman Doktor Faustus selbst als Labor erscheinen lassen. Zeitblom betont, das „eigentlich[e] Kernstück der Suite“ (DF: 461) Leverkühns sei nicht der oben geschilderte Text, sondern der zweite, nämlich der Text mit dem Titel „Von der Geburt des seligen Papstes Gregor“ (DF: 461). Dieser handelt von einem doppelten Inzest: Aus der Liebe eines ,königlichen Geschwisterpaars“ (DF: 462) wird ein Kind geboren, das die Mutter, ,nicht ohne ein unterrichtendes Schrifttäfelchen sowie Gold und Silber für seine Auferziehung [...] den Meereswogen" (ebd.) übergibt. Ein Abt, der ein Kloster leitet, findet das Kind, tauft es auf den Namen Gregor und kümmert sich um seine Erziehung. Gregors Mutter weigert sich, teils aus Treue dem Bruder gegenüber teils, weil sie sich ,als eine Entweihte, der christlichen Ehe Unwürdige betrachtet“ (ebd.), einen Mann zu heiraten. Dies provoziert den Zorn eines „Herzog[s] des Auslandes“ (ebd.), der als Folge ihrer Ablehnung ihr ganzes Reich erobert. Gregor, der mittlerweile das Kloster verlassen hat und sich auf dem Weg zum Heiligen Grab befindet, kommt jedoch zu Hilfe und befreit das ganze Reich; Gregors Mutter erklärt sich bereit,

\footnotetext{
${ }^{35}$ Vgl. DF: 459.

${ }^{36}$ Vgl. DF: 466 f.: ,[...] und Schwerdtfeger, in entfesselter Zutraulichkeit, nahm die Lizenz des Augenblicks wahr, indem er mit einem ,Das hast du großartig gemacht! Adrian umarmte und dessen Kopf an den seinen drückte. Ich sah Rüdigers [Schildknapp] ohnedies schon bitterlichen Mund sich mißbilligend verziehen und konnte selbst nicht umhin, ein ,Genug!‘ zu murmeln und die Hand auszustrecken, wie um den Hemmungslosen, Distanzvergessenen zurückzuholen“.
} 
ihn zu heiraten. Sobald Gregor herausfindet, wen er geheiratet hat, büßt er siebzehn Jahre lang seine Sünde ab: Ein Fischer fährt ihn ,zu einem flutumbrandeten Felsen“ (DF: 464) in der See, legt Fesseln an seine Füße und wirft den Schlüssel ins Meer. Inzwischen stirbt der Papst in Rom und eine Stimme aus dem Himmel befiehlt, nach einem Mann namens Gregor zu suchen. So wird er zum Papst Gregor ernannt. Seine Mutter, die mittlerweile viel Gutes über den neuen Papst gehört hat, macht sich zum Zweck der Sündenerlösung auf den Weg nach Rom: Als sich die beiden wiedersehen, begreifen sie, dass Gott ihnen verziehen hat. In einem vom Gregor gestifteten Kloster lebt die Mutter als Äbtissin bis zu ihrem Tod.

Ein zentrales Motiv des Textes ist zweifelsohne das des Inzests, das wie im Fall von Ödipus oder der Antigone in einen mythischen Kontext eingebettet wird. Die Behandlung des Motivs stellt keinen Einzelfall in Thomas Manns Gesamtwerk dar. In den folgenden Unterabschnitten wird dies anhand der Novelle Wälsungenblut, der Gesta-Kapitel von Doktor Faustus sowie des Romans Der Erwählte chronologisch rekonstruiert. Dies soll Kontinuitäten und Veränderungen im Rahmen dieses Prozesses, also jenes Braidotti zufolge „experimenting with transformations“" 37 erfassbar machen.

\subsubsection{Das Motiv des Inzests in Wälsungenblut}

Als erste Etappe von Thomas Manns Arbeit am Motiv des Inzests sei hier die Novelle Wälsungenblut (1905) ${ }^{38}$ erwähnt, die Wagnerrezeption ${ }^{39}$ und Décadence kombiniert. ${ }^{40}$ Hauptfiguren dieser Novelle sind die Zwillinge Siegmund und Sieglind Aarenhold, Kinder eines reichen jüdischen Privatmannes, die einander sehr

\footnotetext{
${ }^{37}$ Braidotti: Intensive Genre, S. 48.

${ }^{38}$ Mann, T.: Wälsungenblut. In: Ders.: Frühe Erzählungen. 1893-1912. Hrsg. v. Terence J. Reed unter Mitarbeit v. Malte Herwig. GkFA Bd. 2.1, S. 429-463.

${ }^{39} \mathrm{Zu}$ Wagners Vorlage siehe Gottwald, Herwig: Das Inzest-Motiv bei Richard Wagner und Thomas Mann. In: Weichselbaum, Hans (Hrsg.): Androgynie und Inzest in der Literatur um 1900. Salzburg/Wien: Müller 2005, S. 181-203 (insb. S. 186-193).

${ }^{40}$ Siehe Schoene, Anja: „Ach, wäre fern, was ich liebe!“ Studien zur Inzestthematik in der Literatur der Jahrhundertwende. Würzburg: Königshausen \& Neumann 1997, S. 112; Marx, Friedhelm: Inzest. Grenze und Grenzüberschreitung bei Ulrike Draesner und Thomas Mann. In: Catani, Stephanie u. Friedhelm Marx (Hrsg.): Familien, Geschlechter, Macht: Beziehungen im Werk Ulrike Draesners. Göttingen: Wallstein, S. 61-73 (insb. S. 62-70).
} 
nah stehen. Auslöser der ersten inzestuösen Beziehung ist der Besuch der beiden Zwillinge einer Vorstellung von Wagners Die Walküre: Die Oper handelt von der Liebe der gleichnamigen germanischen Götterkinder Siegmund und Sieglind. Die Zwillinge besuchen die Veranstaltung am Tag vor Sieglinds Hochzeit mit von Beckerath, der ,im Ministerium tätig und von Familie“411 ist. Die Novelle endet gleich nach der inzestuösen Beziehung und lässt die Frage unbeantwortet, was nun mit der Hochzeit von Sieglind passieren wird. Der Inzest der jüdischen Zwillinge Aarenhold ist laut Schoene, ,im Vergleich zu Wagners mythisch gedachtem eine Parodie“: ${ }^{42}$ Der Mythos als Modell übernehme keine erlösende Funktion in Wälsungenblut mehr, vielmehr werde in der Novelle die Krankheit der damaligen Zeit diagnostiziert, nämlich ,die Unfähigkeit, über narzißstische Liebe hinweg zum Anderen zu finden". ${ }^{43}$ Schoene hebt gleichzeitig hervor, wie eng die Problematik des Inzests in der Literatur um die Jahrhundertwende, der sich ihre Studie widmet, „mit dem Problem der Rassenzugehörigkeit, speziell des Judentums“"44 verquickt sei: Das schlägt sich in Thomas Manns Novelle nieder, die die Forscherin als eine der ersten Auseinandersetzungen mit der (gefährlichen) Macht von Mythen betrachtet. Dies scheint Roland Barthes Ausführungen in Mythen des Alltags unter vielen Aspekten zu antizipieren. ${ }^{45}$ Roland Barthes und Thomas Mann haben laut Schoene auch gemein, dass sie den Mythos als Metasprache gelesen haben, ,deren Form sich unablässig mit dem Sinn der ,Objektsprache ' auffüllt" “ ${ }^{46}$ Dieser Auffassung zufolge besteht eine der Haupteigenschaften eines Mythos darin, angepasst und bearbeitet zu werden, damit er stets an Bedeutungen gewinnt. ${ }^{47}$ Merkmale dieser ersten Etappe der Arbeit am Motiv des Inzests im Schaffen Thomas Manns sind daher: die Wagnerrezeption, die Rassenfrage, die in Wälsungenblut mit dem Judentum in Verbindung gebracht wird, die Lektüre des Mythos durch die Parodie und die Anerkennung einer Gefährlichkeit des Mythos sowie die Frage nach der Liebesfähigkeit. In Wälsungenblut kommt nur der Geschwisterinzest zum Tragen, bei dem die Geschlechterdifferenz im Vordergrund steht. ${ }^{48}$

\footnotetext{
${ }^{41}$ Mann: Wälsungenblut, S. 445.

${ }^{42}$ Schoene: „Ach, wäre fern, was ich liebe!“, S. 113

${ }^{43}$ Ebd., S. 116. Schoene demonstriert in ihrer Studie, wie eng die Themenfelder Inzest, Narzissmus und Androgynie verknüpft sind. Vgl. ebd., S. 11.

${ }^{44}$ Ebd., S. 221.

${ }^{45}$ Schoene: „Ach, wäre fern, was ich liebe!“, S. 222 (auch Fußnote 110); Barthes, Roland: Mythen des Alltags. Übers. v. Horst Brühmann. Berlin: Suhrkamp 2016 [Paris 1957], 4. Aufl. Vgl. auch Kap. 9.

${ }^{46}$ Ebd., S. 117.

${ }^{47}$ Vgl. Kap. 9.

${ }^{48}$ Siehe ebd., S. 10.
} 


\subsubsection{Das Motiv des Inzests in Doktor Faustus}

In Doktor Faustus taucht das Motiv des Inzests sowohl in der Form des Geschwisterinzests als auch in der Form des Eltern-Kind-Inzests auf; bei diesem letzten Typ liegt der Fokus auf dem „Generationsunterschied, wobei beim Elternteil der Aspekt der Verjüngung, beim Kind der der Regression besonders hervortritt" 49 Dass die Rassenfrage immer noch eine Rolle spielt, ${ }^{50}$ bestätigt bereits der Titel der Exempelsammlung, Gesta romanorum, der gleichzeitig verdeutlicht, dass es sich hier um Taten handelt, die dem historisch-geographischen Raum des Römischen Reiches zuzuordnen sind. Eher als das Judentum wird hier das Christentum thematisiert, was anhand von Handlungen, Figuren und der moralischen Absicht des Werkes deutlich wird. Außerdem korrespondiert die Zeit der Intradiegese mit den ersten Jahren des Ersten Weltkriegs: Bevor Zeitblom auf Leverkühns Komposition zu sprechen kommt, berichtet er von den damaligen ,,volkstümlichen Hochgefühlen“ (DF: 436), von dem Überlegenheitsgefühl Deutschlands, das sich als zum Krieg gezwungenes Volk begreift, das durch den Krieg den Durchbruch „,zur dominierenden Weltmacht“ (DF: 402) erreichen möchte. Die Frage nach der Liebesfähigkeit ist eine, die auch Leverkühns Vertonung prägt. Dies geht etwa aus der folgenden Auflistung Zeitbloms der ,,affektbeladenen Höhepunkte“ (DF: 463) der Handlung der Vorlage hervor, also der Stellen in der Narration, wo Pathos aufgebaut wird, und ,die in der Puppenoper auf so wunderlich-wunderbare Weise zu ihrem Rechte kommen“" (ebd.):

[W]enn sie bei der Nachricht vom Tode des verbrecherisch Erkannten in die merkwürdige Klage ausbricht: „Dahin ist meine Hoffnung, dahin ist meine Kraft, mein einziger Bruder, mein zweites Ich!“ [...] Oder wenn sie, da sie gewahr wird, mit wem sie in zärtlichster Ehe lebt, zu ihm spricht: „O mein süßer Sohn, du bist mein einziges Kind, du bist mein Mann und mein Herr, du bist mein und meines Bruders Sohn, o mein süßes Kind, und du mein Gott, warum hast du mich lassen geboren werden!“ (DF: $463 \mathrm{f}$.)

Um die Analyse der Behandlung des Motivs des Inzests im Gesamtwerk Thomas Manns fortsetzen zu können, gilt es nun, diesen Aspekt in Doktor Faustus,

\footnotetext{
${ }^{49}$ Ebd., S. 9. Zu bemerken ist hier, dass der Aspekt der Regression nicht nur durch Zeitbloms Wertungen des musikalischen Stils der Suite Leverkühns, sondern auch, metadiegetisch, eben durch den Eltern-Kind-Inzest von Gregor mit seiner Mutter thematisiert wird.

${ }^{50}$ Gottwald spricht von „literarische[r] Geschichte der Blut-Mythologeme“, die ,,von Goethes Faust bis Thomas Manns Doktor Faustus und Hans Henny Jahnns Fluß ohne Ufer“ reicht. Gottwald: Das Inzest-Motiv, S. 192.
} 
der im Mittelpunkt der vorliegenden Studie steht, näher zu beleuchten. Als guten Ausgangspunkt bietet sich das oben erwähnte Zitat aus dem Roman, weil dort schon viele relevante Aspekte genannt werden. Zunächst drückt die zweimalige Verwendung des Adjektivs, einzig' die Einzigartigkeit des inzestuösen Verhältnisses aus: Dem Inzest liegt ein Spannungsverhältnis zwischen Auserwähltheit und Tabu zugrunde. ${ }^{51}$ Letzteres kommt im obigen Zitat durch die verzweifelte Hinwendung von Gregors Mutter an Gott zum Ausdruck, der in spätmittelalterlichen Gesellschaften als letzte verbietende Instanz gilt und dem der Ursprung des Tabus selbst attribuiert wird. Durch die Worte „mein zweites Ich“ tritt das Motiv des Narzissmus und „des Wunsches nach Ich-Integration“52 zutage; die Fülle an Attributen, mit denen der jeweilige Partner durch die rhetorische $a c c u$ mulatio bezeichnet wird (Bruder, zweites Ich bzw. Mann, Herr, Bruders Sohn, Kind), verweist wie im Fall des Mythos der Antigone, dem sich Butler widmet, auf ,deformation and displacement“53 von Verwandtschaft, die vom Inzest verursacht werden. Diese Attribute werden als Synonyme behandelt, was eine gewisse ,interchangeability“54 zwischen Familienmitgliedern ausdrückt.

Adrian Leverkühns fiktive Vertonung von Gregors Exempel aus den Gesta romanorum widmet sich, wie bereits erwähnt, nicht nur einer Form des Inzests, sondern zwei unterschiedlichen Formen, die dazu noch unterschiedlich vollzogen werden. Die erste Form ist die des Geschwisterinzests, der bewusst von Gregors Mutter und ihrem Bruder vollzogen wird und auch Fortpflanzung einschließt, da Gregor geboren wird. Der Geschwisterinzest provoziert eine Deformation

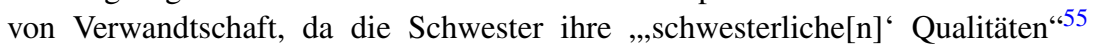
und der Bruder seine „,brüderlichen“ verliert, und so eine Deplatzierung in ein anderes System bewirkt, nämlich in das des sexuellen Begehrens, in dem die Schwester zur geliebten Frau und der Bruder zum geliebten Mann wird. ${ }^{56}$ Das Verwandtschaftssystem wird dadurch jedoch nicht völlig unwirksam: Gregors Mutter spricht von ihrem Bruder ,als zweites Ich“ und betont somit den narzisstischen Charakter (nicht nur) des Geschwisterinzests einerseits und andererseits den Wunsch nach einer extrem endogamischen und exklusiven Liebesbeziehung ${ }^{57}$

\footnotetext{
${ }^{51}$ Ebd.

${ }^{52}$ Frank: Inzest und Autor-Imago, S. 53.

${ }^{53}$ Butler: Antigone's Claim, S. 24.

${ }^{54}$ Ebd., S. 61.

${ }^{55}$ Schoene: „Ach, wäre fern, was ich liebe!“”, S. 11.

${ }^{56}$ Ebd.

${ }^{57}$ Auch aus diesem Grund verknüpft sich der Inzest als ,refusal by both partners to share their common genetic endowment with outsiders" mit der Rassenfrage. Hoelzel, Alfred:
} 
außerhalb der „,cultural intellegibility“, 58 in der Geschlechterdifferenz teils markiert (Schwester/Bruder, Mann/Frau), teils aufgehoben wird. Bei der Aufhebung der Geschlechterdifferenz stellen sich Fragen, wie: Welches Geschlecht soll denn dieses zweite Ich haben? Ist das überhaupt von Bedeutung? Deleuze und Guattari definieren diese Art von Inzest als „Schizo-Inzest“, 59 der deterritorialisierend ist, da die Schwester nicht die Mutter ersetze ${ }^{60}$ sondern ,auf der anderen Seite des Klassenkampfs steht, auf der Seite der Dienstmädchen und der Huren“. 61

Die zweite Form von Inzest, die in Gregors Mythos auftaucht, ist die des Eltern-Kind-Inzests, der jedoch unbewusst vollzogen wird: Mythischer Vorläufer ist bekanntlich Ödipus, dem sich Freud gewidmet und den er in seine Theorie der sexuellen Entwicklung des männlichen Kindes eingebettet hat. ${ }^{62}$ Das Modell, das sich laut Schoene von Freuds Theorie ableiten lässt, ist ,die Reproduktion des patriarchalen Systems durch den ,Ödipus ““.63 Auch Deleuze und Guattari sprechen im Fall des Ödipus-Inzests nicht von Deterritorialisierung, sondern von einer Reterritorialisierung, da der Inzest ,mit der Mutter [...], also mit einer Territorialität ${ }^{\star 64}$ vollzogen wird. Das patriarchale System wird auch in Gregors Mythos reproduziert, da Gregor schließlich sogar Papst, also zur machtlegitimierenden Instanz des politisch-gesellschaftlichen Systems der damaligen Zeit selbst wird. Seine Mutter wird durch seine Entscheidung Äbtissin; beide erhalten erneut eine privilegierte Stellung innerhalb des sozialen Systems, die sogar noch höher ist als die vorherige.

Da aber Leverkühns Vertonung auf Parodie beruht, gilt es, die Frage aufzuwerfen, ob es angebracht ist, auch bezüglich Leverkühns sekundären, fiktiven intermedialen Produkts von Reterritorialisierung zu sprechen. Die Wirkung der Parodie auf einer fiktiven discours-spezifischen Ebene ist die Infragestellung der Vorlage - und das gilt auch, weiter gefasst, für das intramediale Verhältnis

Leverkühn, The Mermaid and Echo: A Tale of Faustian Incest. In: Symposium 42 (1988) H. 1, S. 3-16, hier: S. 11.

${ }^{58}$ Butler: Antigone's Claim, S. 54.

${ }^{59}$ Deleuze, Gilles u. Félix Guattari: Kafka. Für eine kleine Literatur. Frankfurt am Main: Suhrkamp 1976 [Paris 1975], S. 92.

${ }^{60}$ Dieser Auffassung ist z. B. Freud. Siehe Schoene: „Ach, wäre fern, was ich liebe!“, S. 19.

${ }^{61}$ Deleuze u. Guattari: Kafka, ebd.

${ }^{62}$ Schoene: „Ach, wäre fern, was ich liebe!“, S. 19; vgl. Freud, Sigmund: Aus der Geschichte einer infantilen Neurose. Leipzig (u. a.): Internationaler Psychoanalytischer Verlag 1924.

${ }^{63}$ Ebd., S. 19

${ }^{64}$ Deleuze u. Guattari: Kafka, ebd., Herv. i. O. 
zwischen der spätmittelalterlichen Exempelsammlung und Doktor Faustus. Die Vorlage wird dekonstruiert und ebenfalls deterritorialisiert: Während die Intention des spätmittelalterlichen Textes moralischer Natur war, ist die Absicht von Leverkühns Vertonung das genaue Gegenteil, eben jene moralische Intention zu demaskieren, unwirksam zu machen und für lächerlich zu erklären.

Bereits aus der Analyse dieser zweiten Etappe der Arbeit Manns am Motiv des Inzests in einem mythischen Kontext lassen sich Kontinuitäten und Differenzen feststellen. ${ }^{65}$ Gemeinsamkeiten betreffen etwa den Rückgriff auf die Parodie und die Verknüpfung des Inzest-Motivs mit der historisch-politischen Situation, folglich auch mit der Rassenfrage. Ein Unterschied, der eher eine Ergänzung ist, stellt die Behandlung zweier Typen von Inzest dar, die Implikationen für die Darstellung des Verwandtschaftssystems im Text hat.

\subsubsection{Das Motiv des Inzests in Der Erwählte}

Um diesen Prozess weiterzuverfolgen, muss man als letzte Etappe den Roman Der Erwählte (1951) in die Analyse einbeziehen. Der paratextuelle Hinweis des Titels drückt unmittelbar und explizit aus, was vorher bezüglich der Adaption des Textes aus den Gesta über Papst Gregor bereits konstatiert wurde: Durch den Inzest, die Anerkennung der Schuld und die darauf folgende Askese wird Gregor zum Erwählten. So Schoene: ${ }^{66}$

Will das Individuum sich dennoch behaupten, muß es zum Dieb an der eigenen „Präexistenz“ werden. Daß der Weg tatsächlich über den Inzest führen muß, bestätigt fast fünfzig Jahre später ${ }^{67}$ Der Erwählte, dessen Schuld schließlich doch zur Erfüllung führt.

Schoene verdeutlicht in diesem Zitat, was Gregor zum Erwählten macht. Auch in der Entstehung des „Doktor Faustus “ erörtert Thomas Mann: „Extreme Sündhaftigkeit, extreme Buße, nur diese Abfolge schafft Heiligkeit“ (Ent: 114). Ob im

\footnotetext{
${ }^{65}$ Hoelzel betrachtet das Inzest-Motiv als wiederkehrendes Motiv von Doktor Faustus, was auch bei Leverkühns Abschiedsrede vorkommt: Der Komponist bezeichnet Echo in seinem Delirium als seinen Sohn mit der „kleine[n] Seejungfrau“ (DF: 724), die wiederum seine „Schwester und süße Braut“ (ebd.; Herv. A. O.) sei. Siehe Hoelzel: Leverkühn, The Mermaid and Echo.

${ }^{66}$ Schoene: „Ach, wäre fern, was ich liebe!“, S. 117.

${ }^{67}$ Schoene nimmt hier auf die Novelle Wälsungenblut Bezug.
} 
Fall von Der Erwählte daher von einem Inzestprivileg gesprochen werden kann, ${ }^{68}$ erscheint in dieser Hinsicht fragwürdig, weil Gregor nicht wirklich dank des Inzests, sondern eher dank einer nach Thomas Manns Worten ,siebzehnjährige[n] unglaubliche[n] Askese" (Ent: 114), also dank der Buße und der Reterritorialisierung, zum Papst berufen wird. ${ }^{69}$ Dieser Aspekt der Buße und der Gnade ist in Doktor Faustus bereits vorhanden, jedoch kann er aus zwei Hauptgründen übersehen werden. Erstens, weil Gregors Mythos nur eine metadiegetische Erzählung im Rahmen von Doktor Faustus ist, also quantitativ betrachtet viel kürzer als in Der Erwählte ist und dazu dient, die Haupthandlung um den Komponisten Leverkühn zu unterstützen und nicht sie zu ersetzen. Zweitens, weil die unzuverlässige Erzählinstanz „die affektbeladenen Höhepunkte“ (DF: 463) des Inzests bei der Beschreibung der fiktiven Vertonung Leverkühns unterstreicht und daher eher erotische als spirituelle Aspekte in den Vordergrund rückt. Zudem lautet der Titel des Werkes in Doktor Faustus: „Von der Geburt des seligen Papstes Gregor“ (DF: 462), während das spätmittelalterliche Exempel den Titel Von der wundersamen Gnade Gottes und der Geburt des seligen Papstes Gregor trägt: ${ }^{70}$ In Leverkühns fiktiver Vertonung nimmt die religiös-moralische Absicht der Vorlage bereits im Titel wenig Platz ein und es wird nur Gregors Seligkeit erwähnt. Aus diesem Grund überrascht es daher nicht, dass viele Faustus-Interpretationen den Aspekt der Gnade für nachranging ansehen. ${ }^{71}$

Nicht nur die Erfüllung steht bei Der Erwählte im Vordergrund, sondern auch die parodistische Adaption der spätmittelalterlichen Vorlage. Bei Leverkühn erfährt die Leser*innenschaft indirekt durch Zeitbloms Wiedergabe, dass die Vorlage in der Komposition einer parodistischen Revision unterzogen wird. Die Erzählinstanz spricht beispielsweise von der Umwertung der ursprünglichen didaktisch-moralischen Intention des Textes ins Skurrile und Erotische, was das Medium des Puppenspiels unterstützt haben soll: Es handelt sich aber um die im

${ }^{68}$ Dieser Auffassung ist beispielsweise Svenja Frank. Siehe Frank: Inzest und Autor-Imago, S. 56 Fußnote 16.

${ }^{69}$ Obwohl sich der Inzest bekanntlich in einem Spannungsverhältnis zwischen Auserwähltheit und Kulturtabu befindet.

${ }^{70}$ Siehe Gesta Romanorum: S. 141.

${ }^{71}$ Hoelzel sieht etwa in der Behandlung des Mythos Gregors in Doktor Faustus im Vergleich zu Der Erwählte keine ,redeeming features“, weil der Inzest als ,refusal by both partners to share their common genetic endowment with outsiders" nur auf ,repulsive aspects of National-Socialist Germany and its origins" und auf Leverkühns Persönlichkeit zurückzuführen sei. Hoelzel: Leverkühn, The Mermaid and Echo, S. 11 u. 15. Siehe auch Hermanns: Thomas Manns Roman, S. 37. Murdoch vergleicht Leverkühn und Gregor und kommt zu der Schlussfolgerung, dass trotz ähnlicher Biographien doch nur Gregor Erlösung zuteil wird. Murdoch: Gregorius, S. 201. 
Vergleich zur Apocalipsis oder Weheklag nicht besonders ausführliche Beschreibung einer fiktiven Komposition, die sich die Leser*innen durch das intermediale telling nur vorstellen können. Bei Der Erwählte bildet Gregors Mythos den Kern des Romans und es lässt sich nicht bezweifeln, dass es sich hier um eine parodistische Lektüre der Gesta romanorum handelt, da die Leser*innenschaft mit der Umsetzung der Adaption direkt konfrontiert wird. Die Präsenz eines Erzählers, der sich als Mönch schämt, von bestimmten Stellen zu erzählen, ${ }^{72}$ und der sich für „,die Inkarnation des Geistes der Erzählung“73 hält, verstärkt etwa den parodistischen Aspekt. ${ }^{74}$

Der Roman Doktor Faustus kann daher als Labor für den Roman Der Erwählte gesehen werden, als experimentiervolle Transitionsphase in Thomas Manns langjähriger Auseinandersetzung mit dem Motiv des Inzests in einem mythischen Kontext. Ob parodistisch oder nicht parodistisch gefärbt, das Motiv des Inzests wirft unweigerlich die Frage „,nach Alternativen der bestehenden Ordnung“ "75 nach der „Möglichkeit einer anderen Existenzweise außerhalb des dominierenden hierarchisch-patriarchalen Systems“76 auf. Diese Frage wird im literarischen Text etwa durch die Figur von Antigone oder Gregors Mutter und ihre Deterritorialisierungsprozesse, die gesellschaftliche Verhältnisse subvertieren, ${ }^{77}$ transportiert: Sie entspricht jener ,representational quality of narratives“ ${ }^{78}$ also der Fähigkeit narrativer Texte, mögliche Welten zu entwerfen. Die Frage nach Alternativen des bestehenden Verwandtschafts- und Begehrenssystems prägt in Doktor Faustus nicht nur Leverkühns Adaption der Gesta, sie taucht auch in der (platonischen?) homosexuellen Beziehung zwischen Schwerdtfeger und Leverkühn, dem Liebesverbot des Teufels und im Ehebruch Ines Institoris mit Schwerdtfeger auf: Es

\footnotetext{
${ }^{72}$ Siehe etwa Mann: Der Erwählte, S. 24: ,[...] [F]erner weil ich, die eigenen Augen mönchisch niederschlagend, berichte $[\ldots]^{\text {“. }}$.

${ }^{73}$ Ebd., S. 8.

${ }^{74}$ Dies öffnet die Tür für literaturwissenschaftliche Untersuchungen zu metanarrativen Verfahren. Außerdem würde sich lohnen zu untersuchen, ob der Mönch-Erzähler von Der Erwählte für eine zuverlässige Erzählinstanz zu halten ist. In dieser Hinsicht wäre auch die Arbeit an der unzuverlässigen Erzählinstanz Zeitblom als Experimentieren und Vorbereitung auf das später veröffentlichte Werk anzusehen.

${ }^{75}$ Schoene: „Ach, wäre fern, was ich liebe!“, S. 21.

${ }^{76}$ Ebd., S. 23.

${ }^{77}$ Siehe ebd., S. 229. Was die gesellschaftlichen Verhältnisse angeht, so unterstreicht Schoene zurecht, dass diese in ,ihrer historischen Bedingtheit zu erfassen und zu untersuchen" sind. Ebd., S. 12.

${ }^{78}$ Wolf: Narrativity in Instrumental Music?, S. 485, Herv. i. O.
} 
wundert nicht, dass der einzige Reterritorialisierungsversuch Leverkühns durch den seltsamen Heiratsantrag an Marie Godeau zum Scheitern verurteilt ist.

Die zwei Kapitel über die Gesta romanorum machen jenes ,,experimenting with transformations "79 aus textimmanenter und intramedial-diachroner, die Werke desselben Autors berücksichtigender Perspektive sichtbar, dementsprechend sowohl wenn sich die Analyse nur auf die Gesta-Kapitel von Doktor Faustus konzentriert als auch wenn sie chronologisch Manns Arbeit am Inzest-Motiv in den Werken Wälsungenblut, Doktor Faustus und Der Erwählte rekonstruiert. Das Hauptanliegen des darauf folgenden Abschnitts besteht darin zu untersuchen, wie einige Mikroformen der Kapitel anhand der Analyse zweier intermedialer Transpositionen in das Medium der Musik transferiert und transformiert werden.

\subsection{Vom Roman zur Musik}

Im Folgenden werden zwei Kompositionen analysiert, die als Vorlage die GestaKapitel von Doktor Faustus wählen, nämlich Beyers Die Gesta romanorum Musik des Adrian Leverkühn und Odegards The Calling of St. Gregory. Es soll aus intermedialer Perspektive untersucht werden, wie die Hauptaspekte von Leverkühns Puppenspiel, z. B. das Inzest-Motiv oder die Umwertung der moralischen Absicht der Vorlage u. a. durch die Parodie sowie das Ziel einer größeren Zugänglichkeit des Werkes, im Medium der Musik Umsetzung finden. Daneben wird auch der Frage nachgegangen, was die jeweilige Transposition über die Rezeption der Kapitel aussagt. Um diese Frage zu beantworten, wird sich die Analyse nicht nur auf die Transfers und Transformationen aus Doktor Faustus, sondern auch auf spezifische Eigenschaften der jeweiligen Komposition konzentrieren.

\subsubsection{Zwischen Rekonstruktion und Adaption: Frank Michael Beyers Die Gesta romanorum - Musik des Adrian Leverkühn}

Die Gesta romanorum - Musik des Adrian Leverkühn ist eine Komposition von Frank Michael Beyer (1928-2008), einem fast das ganze Leben lang in Berlin tätigen Komponisten. ${ }^{80}$ Sie wurde für zwei Anlässe adaptiert: zuerst für eine

\footnotetext{
${ }^{79}$ Braidotti: Intensive Genre, S. 48.

${ }^{80}$ Zum Komponisten siehe: Stahl, Claudia: Art. Beyer, Frank Michael. In: MGG Online. Veröffentlicht 2016.<https://www.mgg-online.com/mgg/stable/51381> (letzter Zugriff:
} 
Fernsehsendung Alexander Kluges, 10 vor 11, die am 8. April 1991 auf RTL plus übertragen wurde, und dann für ein Konzert, das am 3. November $1996 \mathrm{im}$ Berliner Konzerthaus stattfand. ${ }^{81}$ Die Fernsehfassung, die am 22. Mai 1990 die Münchner Philharmoniker aufnahmen, ist für Kammerensemble gedacht. Die TVProduktion kombiniert Musik, Film und Erklärungen von Kluge und Beyer. Die Version für das Konzerthaus sieht wie in Leverkühns Komposition ${ }^{82}$ die Präsenz eines Sprechers - in dem Fall Daniel Minetti - vor.

Im Folgenden wird auf Kontinuitäten und Differenzen vor allem zwischen Beyers Vertonung und den Gesta-Kapiteln aus Doktor Faustus eingegangen. Auffällig ist zunächst einmal, dass sich Beyer auf die Geschichte Gregors konzentriert: Es handelt sich daher um eine partielle Vertonung nicht nur des Romans selbst, sondern auch des fiktiven Werkes Leverkühns. Im Autograf findet man neben dem Stück „Gregorius“ auch drei weitere Stücke: eins davon (S. 6-9), das den Titel „Löwe und Löwin - Vom Ehebruch“ trägt, bezieht sich sehr wahrscheinlich auf die erste von Leverkühn vertonte Geschichte aus den Gesta, da es dem Titel entsprechend von einem ehebrecherischen Liebesverhältnis handelt. Im Rahmen der Fernsehsendung wurde dieses Stück aber nicht aufgeführt. Das dritte Stück des Autografs (S. 10-14) trägt den Titel „Eisenmusik“, das zweite (S. 2), das sich in der Partitur vor dem ersten, „Löwe und Löwin“, befindet, trägt keinen Titel; des Weiteren sind auf der ersten Seite des Autografs kurze musikalische Motive zu finden, die wahrscheinlich als musikalische Untermalung oder musikalische Kommentare oder auch Verbindungselemente für die Fernsehsendung bzw. für die Aufführung im Konzerthaus gedacht wurden, da die Überschrift „,Signale Rite Momente" lautet: Diese sind zum Teil auch in unterschiedlichen Besetzungen aufführbar. „Gregorius“ ist das vierte Stück des Autografs (S. 15-21). Die folgende Analyse beruht vor allem gleichzeitig auf dem undatierten Autograf und auf der Fernsehsendung. ${ }^{83}$

Ein zweiter erwähnenswerter Aspekt von Beyers Gesta besteht darin, dass diese Komposition weder in der Fassung für die Fernsehsendung noch in der für das Konzerthaus ein Puppenspiel ist: Beyer orientiert sich nicht an der Form von Leverkühns Werk. In einem Interview mit Werner Grünzweig, dem Leiter des

21.08.2020); Grünzweig, Werner u. Daniela Reinhold (Hrsg.): Frank Michael Beyer. Hofheim: Wolke 1998.

${ }^{81}$ Das Autograf kann an der Akademie der Künste in Berlin eingesehen werden (Frank Michael Beyer - Archiv 213-215); die Videokassette mit der Sendung befindet sich u. a. in der Bibliothek der Universität der Künste in Berlin (Mediathek WF 1589).

${ }^{82}$ Vgl. DF: 461.

${ }^{83}$ Keine Aufnahme der Version für das Konzerthaus Berlin konnte gefunden werden. 
Musikarchivs der Akademie der Künste in Berlin, wo sich Beyers Nachlass befindet, und dem Musikwissenschaftler Heribert Henrich erklärt der Komponist, dass

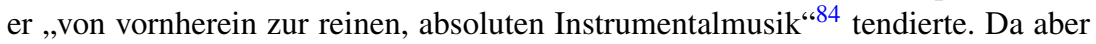
die Komposition als , direct or overt intermediality“ und auch als, intermediale Transposition' einzustufen ist, kann sie für keine reine, absolute Instrumentalmusik gehalten werden. ${ }^{85}$ Die Begründung dafür ist, dass sie eine*n Sprecher*in vorsieht und im Titel sofort auf den Versuch verweist, Thomas Manns Roman zu vertonen. Auch der umstrittene und keineswegs eindeutige Begriff ,Programmusik $^{\circ 66}$ wäre aufgrund des Vorhandenseins eines gesprochenen Textes keine adäquate Bezeichnung; passender scheint das Begriffsinstrumentarium der Intermedialitätsforschung, laut dem es sich um eine Medienkombination handelt. Es werden nämlich das Medium der Musik und das Medium des gesprochenen Wortes und in der Fernsehsendung zusätzlich noch das Medium des Fernsehens miteinander kombiniert. Grundsätzlich könnte auch einfach die Formbezeichnung Suite verwendet werden; in Bezug auf Doktor Faustus kann ebenfalls ausgesagt werden, dass Leverkühns fiktive Vertonung eine Medienkombination und eine Suite ist.

Kontinuitäten lassen sich auch in der Besetzung beobachten: Beyers Stück hat dieselbe Besetzung wie Leverkühns Puppenspiel, die der von Igor Strawinskys Histoire $d u$ soldat entspricht. ${ }^{87}$ Nicht zufällig nimmt dieses Werk Bezug auf den Faust-Mythos: Dies erzeugt einen Loop von intra- und intermedialen Einzelreferenzen, also von Bezügen sowohl auf literarische als auch auf musikalische Adaptionen des Faust-Stoffes. Darüber hinaus realisiert Beyer die Gesta laut Frank Schneider mit ,historischer Authentizität“:88

Behutsam und eindringlich hat Beyer Tendenzen der damaligen Moderne verarbeitet. Die „Zwölftonmusik“ spielte noch keine manifeste Rolle, aber auch radikale „Atonalität“ hätte Leverkühns Konzeption widersprochen. Stattdessen findet sich

${ }^{84}$ „Ich wollte auch nicht Chopin spielen...“. Frank Michael Beyer im Gespräch mit Werner Grünzweig und Heribert Henrich. In: Frank Michael Beyer, S. 64-73, hier: S. 69.

${ }^{85}$ Zum Begriff ,Absolute Musik' siehe etwa: Seidel, Wilhelm: Art. Absolute Musik. In: MGG Online. Zuerst veröffentlicht 1994, online veröffentlicht 2016.<https://www.mggonline.com/mgg/stable/11554> (letzter Zugriff: 21.08.2020).

${ }^{86}$ Dazu siehe etwa Altenburg, Detlef: Art. Programmusik. In: MGG Online. Zuerst veröffentlicht 1997, online veröffentlicht 2016.<https://www.mgg-online.com/mgg/stable/ 12244> (letzter Zugriff: 21.08.2020).

${ }^{87}$ Vgl. die Rezension Frank Schneiders im Konzertheft der Aufführung im Konzerthaus, S. 5. Sie kann an der Akademie der Künste zusammen mit dem Manuskript konsultiert werden (unter derselben Signatur).

${ }^{88}$ Ebd., S. 6. 
eine Nähe zu dem von Mann evozierten Strawinsky, u. a. in der Selbstbezüglichkeit des Werkes mit seinem heraldischen Ankündigungsgestus, der Verwendung ostinater, rhythmisch verschränkter Modelle, dem verfremdenden Rückgriff auf traditionelle Ausdruckstopoi [...].

Auch Albrecht Dümling beschreibt im Tagesspiegel die Musik als eine, die „in meist ruhigen Zeitmaßen kunstreich aus archaischen Formeln im Stile Strawinskys“ ${ }^{89}$ komponiert wurde. Und im Programmheft der Aufführung in Berlin präzisiert der Untertitel: Von der Geburt des seligen Papstes Gregor, nach Adrian Leverkühns Intentionen gestaltet und in Musik gesetzt, für Sprecher und Kammerensemble. ${ }^{90}$ Der paratextuelle Hinweis verdeutlicht die Absicht eines rekonstruktiven Textverständnisses sowie den Versuch, durch die beiden vorhandenen Medien zu erzählen. ${ }^{91}$

Was die Nähe zu Strawinsky angeht, gibt der Komponist selbst zu, von diesem sehr beeinflusst gewesen zu sein: „Anregungen von außen haben eine relativ kleine Rolle gespielt. Die Faszination des Neuen ging zunächst vom Spätwerk Strawinskys aus, das mich bis heute begeistert“. ${ }^{92}$ Frank Michael Beyer äußert zeit seines Lebens keine Faszination für die Darmstädter Avantgarde, also für das, was als das Neue seiner Zeit in Sachen Musik angesehen werden könnte, und wendet sich stattdessen dem Spätwerk Strawinskys zu. ${ }^{93}$ Da er Anfang der 1990er Jahre für das Fernsehen mit Alexander Kluge zusammenarbeitete (die Gesta sind ein Beispiel dafür), könnte man vermuten, dass er neue Formen der Musikvermittlung erproben wollte, um - vergleichbar zu Adrian Leverkühn im Roman - „eine Kunst mit der Menschheit auf du und du“ (DF: 469) zu produzieren. Im Interview stellen ihm Grünzweig und Henrich genau diese Frage, auf die er jedoch

\footnotetext{
${ }^{89}$ Dümling, Albrecht: Kindertrompetenstil. Faustus-Projekt mit dem Kammerensemble Neue Musik Berlin. In: Der Tagesspiegel, 05.09.1996.

${ }^{90}$ Dieser Untertitel erscheint im Autograf nicht; allerdings wurde das Autograf Die Gesta romanorum - Musik des Adrian Leverkühn betitelt.

${ }^{91}$ Siehe Wolf: Narrativity in Instrumental Music?, S. 492. Obwohl Wolf sich hier auf reine Instrumentalmusik bezieht, lassen sich seine Argumentationen zu den ,intracompositional incitements to narrativize" auch auf die analysierte Medienkombination Beyers übertragen. Selbstverständlich gewinnt die Komposition kraft der Präsenz eines*r Sprecher*in an Erzählpotenzial.

${ }^{92}$ Grünzweig u. Henrich: „Ich wollte auch nicht Chopin spielen...“, S. 70. Die Histoire gehört aber nicht zum Spätwerk des Komponisten (Lausanne, 1918).

${ }^{93}$ Siehe ebd.
} 
antwortet, dass er mit Kluge ,nur zusammengearbeitet“94 habe, weil ihm ,das Wechselgespräch mit ihm Freude machte“. ${ }^{95}$ Diesbezüglich führt er aus, dass

[d]ie Frage, welchen Stellenwert heute Musik in der Öffentlichkeit hat, [...] so offen [sei], daß [...] [er] nicht glaube, daß sie sich dort entscheidet, wo es um die Medien geht. Das [...] [sei] vielmehr eine innere Frage, eine Frage dessen, wie die heutige Zeit insgesamt mit der Kultur umgeht. ${ }^{96}$

Abgesehen von der Frage nach dem Stellenwert der Musik in der Öffentlichkeit impliziert das Komponieren der Musik für eine Fernsehsendung, die für keinen elitären Fernsehkanal und in Kooperation mit einem berühmten Moderatoren produziert wurde, die Tatsache, dass das Medienprodukt einem größeren Publikum zur Verfügung gestellt und es nicht für den Konzertsaal komponiert wird. Nicht zuletzt sind diesbezüglich auch die historischen Entstehungsbedingungen des Werkes in die Analyse einzubeziehen, denn 1991 hatte das Fernsehen einen anderen Stellenwert als in der heutigen, von vielfältigen digitalen Medien geprägten Gesellschaft.

Zwar ist Beyers Komposition nicht der Form des Puppenspiels zuzuordnen, greift aber ebenfalls auf ein ,,volksnahes“ Instrument zurück und lässt die Musik mit anderen Medien interagieren, sie experimentiert mit ihnen. Die Art des Mediums, mit dem man auf eine größere Zugänglichkeit des Kunstwerkes abzielt, wird an die jeweilige historische Situation angepasst, was weniger für ,historische[] Authentizität" 97 sondern eher für eine adaptive Lesart spricht. ${ }^{98}$ Selbstverständlich lässt sich das auch als Experiment definieren: Die reine, absolute Musik,

\footnotetext{
${ }^{94}$ Ebd., S. 73. Vgl. auch Koch, Klaus Georg: Kannst du mich komponieren? Keiner weiß, ob das Gute bleibt, wenn niemand es sucht. Ein Gespräch mit dem Komponisten Frank Michael Beyer. In: Berliner Zeitung, 16.06.1999: „Deshalb sollte das Musikleben weitgehend befreit bleiben vom finanziellen Zwang. Wenn es erst einmal soweit gekommen ist, daß etwa die Orchester sagen, wir müssen dieses und jenes realisieren in Hinblick auf den Publikumsbesuch, dann fängt es an mit dem Warencharakter der Musik“.

${ }^{95}$ Ebd.

${ }^{96}$ Ebd.

${ }^{97}$ Schneider: Konzertheft, S. 6 (siehe Fußnote 86).

${ }^{98}$ Siehe dazu auch nochmals das Interview Beyers mit Koch, in dem Beyer die Oper als „Erweiterung der Sprachmöglichkeiten einer Zeit“ definiert. Koch: Kannst du mich komponieren, ebd. Zwar bezieht sich diese Äußerung des Autors auf eine unterschiedliche Musikgattung, spricht aber ebenfalls den Aspekt der Anpassung der musikalischen Mittel an die jeweilige historische Zeit an.
} 
die sonst immerhin unweigerlich von Massenmedien beeinflusst wird, ${ }^{99}$ sucht den Dialog mit dem Medium Fernsehen und mit seinen Akteur*innen. Liest man zudem die Biographie Beyers, so ist festzustellen, dass die Zusammenarbeit mit Kluge kein nur die Gesta betreffender Einzelfall ist: Es handelt sich um ein fortgesetztes Experiment, denn neben dieser Produktion haben die beiden auch noch weitere Folgen von 10 vor 11 zusammen konzipiert. ${ }^{100}$ Auch Beyers Komposition, wie andere (wenn nicht alle) in dieser Studie betrachteten, ${ }^{101}$ ist nicht lediglich „auf dem Papier ausgerechnet“ (PhnM: 20), sondern zieht die Bedingungen der jeweiligen Aufführung (für eine Fernsehsendung, für das Konzerthaus Berlin) in Betracht, indem etwa der Moderator durch eine*n Sprecher*in ersetzt wird (Fassung für das Konzerthaus) oder indem zwischen den einzelnen Stücken weitere Erklärungen geliefert werden (Fassung für die Fernsehsendung).

Nun werden Musik und Struktur des vierten Stückes des Autografs, „Gregorius" näher beleuchtet. Es besteht aus den folgenden vier Teilen:

Teil A - Die Geburt

Teil B - Sein Schicksal

Teil C - Angekettet im Meer

Teil D - Süße Eltern ${ }^{102}$

In Teil A findet man einen Dialog mit Klarinette, Violine und Fagott. Die übrigen Instrumente des Ensembles (Trompete, Posaune, Schlagzeug und Kontrabass) übernehmen hier eine begleitende Funktion bzw. sind nicht zu hören. Der darauf folgende Teil ist dank der ständigen Präsenz des Schlagzeugs rhythmischer gedacht; hier wird vom Schicksal Gregors erzählt. Wie der vorherige ist auch dieser Teil von einer leisen Dynamik gekennzeichnet. Nun musizieren alle Instrumente zusammen. Gegen Ende des zweiten Teils (B) sieht der Komponist die Wiederholung von A ab T. 20 bis zum Fine vor. Teil C ist der längste Teil der Partitur und Teil D schließlich, der den Namen „Süße Eltern“ trägt, ist durch einen Dialog zwischen Klarinette und Fagott charakterisiert (Abbildung 3.1).

Da hier Gregors Mutter den Sohn in Rom besucht, wo er nun Papst ist, wird deutlich, dass diese zwei Instrumente als anthropomorphe Entitäten in einer musikalischen Erzählwelt ${ }^{103}$ jeweils für die beiden Figuren (die Klarinette für Gregor,

\footnotetext{
${ }^{99}$ Siehe Günter, Manuela: Im Vorhof der Kunst. Mediengeschichten der Literatur im 19. Jahrhundert. Bielefeld: transcript 2008 (insb. 2.2).

${ }^{100}$ Siehe Stahl: Beyer; Kreimeier, Klaus: TEN TO ELEVEN oder: Kann man Zeit abbilden? Alexander Kluges Kuriositätenkabinett im Privatfernsehen. In: Die Zeit, 27.11.1992. ${ }^{101}$ Siehe etwa Hagens To Zeitblom (Kap. 7).

${ }^{102}$ Die Titel der einzelnen Teile stammen aus der Fernsehsendung; im Autograf ist nur der Titel „Süße Eltern“ zu finden (S. 20).

${ }^{103}$ Siehe Wolf: Narrativity in Instrumental Music?, S. 494.
} 


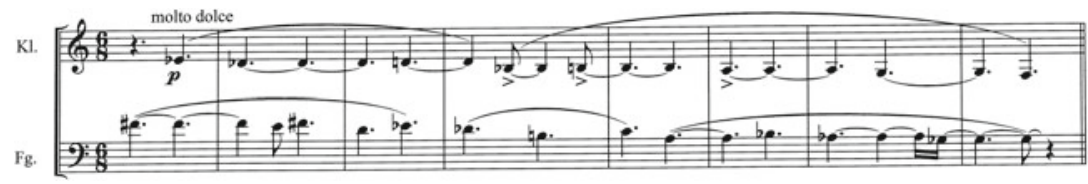

Abbildung 3.1 Klarinette und Fagott in „Süße Eltern“ (T. 5-12)

das Fagott für die Mutter) stehen: Folglich gibt es im Stück kaum Stellen, welche die Präsenz der Klarinette nicht vorsehen. ${ }^{104}$ Insgesamt ist die Musik von Beyers Gesta leise, statisch und eher von Dialogen zwischen Instrumenten als vom Tutti geprägt: Das lässt sich auch dadurch erklären, dass die Musik nicht als einziges Erzählmedium gedacht ist, sondern durch die Moderation ergänzt werden soll, die jene Vagheit des Erzählens in einer rein instrumentalen Form überwindet. ${ }^{105}$ Auch bezüglich Gregors Mythos erweist sich die Vertonung als partiell, da die Handlung an Gregors Geburt ansetzt, sodass der Aspekt des doppelten Inzests und daher der - so Butler - ,,performative repetition“, 106 durch die Verwandtschaft als öffentlichen Skandal wieder eingesetzt wird, im Vergleich zur fiktiven Vertonung Leverkühns weniger Bedeutung zugemessen wird. ${ }^{107}$

Angesichts der Divergenzen zwischen Autograf und Aufführungen treten auch im Fall von Beyers Gesta Experimente und Transformationen zutage, die sich manchmal auch schwer nachverfolgen lassen, zugleich aber deutlich machen, dass das Autograf nicht als Schlussversion zu betrachten ist, sondern Bestandteil eines auf Plurimedialität/Medienhybridisierung und Zugänglichkeit abzielenden Gesamtprozesses ist. Außerdem herrscht in der Komposition ein Spannungsverhältnis zwischen rekonstruktivem und adaptivem Textverständnis: Hinsichtlich der Besetzung, der stilistischen Nähe zu Strawinsky und der Interaktion mit

${ }^{104}$ Auch bei Strawinsky übernimmt das Instrument eine zentrale Funktion: Der Komponist fertigte dann eine Version der Histoire für Klarinette, Violine und Klavier an. Die Violine spielt bei Beyer in Teil A eine wichtige Rolle. Siehe Scherliess, Volker: Igor' Fëdorovič Stravinskij. In: MGG online. Veröffentlicht 2016.<https://www.mgg-online.com/mgg/sta ble/11849> (letzter Zugriff: 21.08.2020).

${ }^{105}$ Siehe Wolf: Narrativity in Instrumental Music?, S. 480.

${ }^{106}$ Butler: Antigone's Claim, S. 58.

${ }^{107}$ Vgl. ebd. Gregors Mythos betreffend lässt sich im Gegensatz zum Mythos der Antigone nicht wirklich vom öffentlichen Skandal sprechen, da nur wenige Figuren etwa vom ersten Geschwisterinzest erfahren. Vgl. etwa Mann, T.: Der Erwählte, S. 37-50. 
volksnahen Medien orientiert sich Beyers Komposition an ihrer Vorlage, nämlich Leverkühns Puppenspiel, während sie sich hinsichtlich des Rückgriffs auf das Medium Fernsehen von ihr distanziert.

\subsection{2 "Entauratisierung des Inzests": ${ }^{108}$ Peter S. Odegards The Calling of St. Gregory}

The Calling of St. Gregory von Peter S. Odegard (1929-2009) ist eine Komposition für zwei Sänger (Bass und Bariton), Flexaton ${ }^{109}$ und Tonband, die 1988 speziell für das Konzert „Music from Dr. Faustus“ an der University of California, Irvine entstand. ${ }^{110}$ Das Konzert fand anlässlich des Symposiums „Dr. Faustus at the Margin of Modernism" statt, das u. a. vom Humanities Research Institute der University of California gefördert wurde. Die Idee des Konzerts war, die Musik im Leben Adrian Leverkühns grob zusammenzufassen und aufzuführen: Es sollten nicht nur seine fiktiven Werke, sondern auch intermediale Einzelreferenzen des Romans, etwa Beethovens Sonate opus 111 und das Volkslied $O$ wie wohl ist mir am Abend in den Konzertsaal geholt werden. Aufgrund von Zeit- und Besetzungsproblemen konnten jedoch die Apocalipsis und die Weheklag, die wahrscheinlich umfangreichsten und ambitioniertesten Projekte von Adrian Leverkühn, nicht vertont werden. Zwei Komponisten und Professoren der Universität, Zelman Bokser und Peter Odegard, entschieden sich jeweils für das 1927

\footnotetext{
${ }^{108}$ Marx: Inzest, S. 68.

${ }^{109}$ Ein Flexaton ist ein Instrument für Spezialeffekte, das aus einer flexiblen Metallplatte besteht, die sich innerhalb eines Drahtrahmens mit Griff und zwei Klöppeln befindet. Schüttelt man das Instrument mit zitternder Bewegung, so werden die Klöppel gegen die Platte geschlagen: Das stärkere oder schwächere Drücken kann den Ton erhöhen oder erniedrigen. Ursprünglich stammt das Instrument aus der Jazz-Musik. Siehe Blade, James u. James Holland: Flexatone. In: Grove Music Online. Zuerst veröffentlicht 20.01.2001, online veröffentlicht 2001.<https://doi.org/10.1093/gmo/9781561592630. article.09829> (letzter Zugriff: 21.08.2020).

${ }^{110}$ Das Konzert wurde von Prof. Dr. Margaret Murata, Professorin für Musikwissenschaft an derselben Universität, organisiert und von Prof. Dr. Herbert Lehnert, Professor an der German School of Humanities ebenfalls der University of California, Irvine, konzipiert. Prof. Murata stellte dankenswerterweise für die hier vorliegende Arbeit alle Materialien zur Verfügung, die zur Beschreibung des Stückes nötig waren, nämlich das Autograf, die Aufnahme der Uraufführung sowie Anmerkungen und Erklärungen von Odegard.
} 
vom fiktiven Komponisten Leverkühn verfasste Streichquartett ${ }^{111}$ und die Gesta Romanorum.

Margaret Murata, Organisatorin des Konzerts, unterstützte sie bei der Wahl des zu vertonenden Stückes und erklärte ihre Entscheidung der Los Angeles Times so: ${ }^{112}$

I want the program to show that the musical descriptions in the novel are real. They're not fantasies. They can be turned into real music [that represents] a period when people were trying to do something new and still communicate.

Hier verdeutlicht Murata, dass sich nicht nur das fiktive Puppenspiel Leverkühns, sondern die musikhistorische Zeit selbst auf der Suche nach Neuem durch Experimente und Kommunikation mit dem Publikum befand.

Eine solche Aufgabe, Zeitbloms Beschreibungen von Leverkühns Werken so präzise wie möglich in Musik umzusetzen, ist alles andere als einfach: ,The challenge for our composers was to write in a style that is not current" ${ }^{\prime 13}$ führte die Professorin in dem Interview weiter aus. Wie löste Odegard seinen Worten zufolge das Problem? ${ }^{114}$

I basically picked a style that I thought was commensurate with what Leverkuehn would have done [...]. [M]y puppet opera is primarily a late 19th-Century style with some areas that may lean toward the 12-tone system.

Die Besetzung von Odegards Puppenspiel entspricht nicht der von Doktor Faustus, ${ }^{115}$ obwohl der Komponist im Autograf die Möglichkeit anbietet, eine

${ }^{111}$ Das Stück von Bokser, Leverkühn 1927, wird in der vorliegenden Arbeit aus zwei Hauptgründen ausgelassen. Erstens handelt es sich um eine reine Instrumentalkomposition, also um verdeckte Intermedialität, was die intermediale Analyse wegen des Rückgriffs auf das einzige Medium der Musik erschwert (vgl. 1.1.5). Zweitens wird der Vergleich mit dem Roman auch dadurch erschwert, dass das Stück auf eine kurze Passage von Doktor Faustus Bezug nimmt, der sich die Forschungsliteratur kaum zuwendet.

${ }^{112}$ Flocken, Corinne A.: Mythical Composer's Work Spurs Esoteric Pieces by UCI Professors. In: Los Angeles Times, 11.3.1988 (Ergänzung i. O.)

${ }^{113}$ Ebd.

${ }^{114}$ Ebd.

${ }^{115}$ Da besteht das „,sehr sparsam besetzte[]“ Orchester aus einer Violine, einem Kontrabass, einer Klarinette, einem Fagott, einer Trompete und einer „Posaune nebst Schlagzeug für einen Mann und dazu einem Glockenapparat“ (DF: 461). Die Komposition Leverkühns sieht außerdem eine*n Sprecher*in vor, ,der gleich dem testis des Oratoriums, 
Orchesterbearbeitung für spätere Aufführungen anzufertigen. ${ }^{116}$ Die Form korrespondiert mit der des Romans: Odegards Werk ist ein Puppenspiel. ${ }^{117}$ Wie Beyers Komposition versucht Odegards The Calling of St. Gregory den musikalischen Stil von Leverkühns Werk und der entsprechenden musikhistorischen Zeit (ca. 1915) zu rekonstruieren. Im Programmheft erscheint Leverkühn sogar als Urheber der Komposition (seine Lebzeiten werden auch angegeben) und Odegard als Bearbeiter (,ed. P. Odegard“), was sich als autofiktionales Verfahren einstufen lässt: Odegard ruft die Fiktion hervor, dass er sich um die Herausgabe von Leverkühns Musik gekümmert habe, und spielt mit den Grenzen zwischen Faktualität und Fiktionalität. ${ }^{118}$ Er verweist somit indirekt auf einen wichtigen Aspekt intermedialen Transponierens: Komponist*innen transferieren und transformieren fiktive Musik in faktuale.

Nun wird auf Struktur, Inhalt und musikalische Faktur von Odegards The Calling of St. Gregory eingegangen. Das Stück dauert ungefähr acht Minuten und ist in zwei Hauptteile gegliedert. Der erste Teil (T. 1-55) fängt mit den in den Erklärungen des Autors genannten „magical chords“119 an, die sich laut diesen ,extracompositional“120 Angaben auf ,the loving and passionate glances of the brother and sister who were Gregory's parents"121 beziehen, also auf das Motiv des Geschwisterinzests, das daher anders als bei Beyer zur Handlung von Odegards Stück gehört (Abbildung 3.2):

$\mathrm{Zu}$ bemerken sind am obigen Notenbeispiel auch einige ,intracompositional incitements to narrativize" ${ }^{122}$ nämlich die Präsenz zweier Stimmen und die Tempoangabe, die Hinweise auf die erzählte Zeit und die Erzählzeit liefert. Im Stück

die Handlung in Rezitativ und Erzählung zusammendrängt“ (ebd.). Vor der Apocalipsis experimentiert daher der Komponist auch mit der Verwendung einer erzählenden Stimme.

${ }^{116}$ Siehe „To Whom it May Concern“, S. 1.

${ }^{117} 1988$ wurde nicht das ganze Werk aufgeführt, sondern nur eine Szene, ein Rezitativ und eine Arie. Ob The Calling nur aus diesen drei Stücken besteht und folglich im Untertitel die Fiktion eines Unvollendetseins des Werkes hervorruft (vergleichbar zur Fiktion einer Bearbeiterfunktion Odegards), bleibt unklar. 1988 wurde außerdem das Werk nicht als Puppenspiel, also ohne Inszenierung und Marionetten, uraufgeführt.

${ }^{118}$ Vgl. Zipfel, Frank: Autofiktion. Zwischen den Grenzen von Faktualität, Fiktionalität und Literarität? In: Winko, Simone, Fotis Jannidis u. Gerhard Lauer (Hrsg.): Grenzen der Literatur. Zu Begriff und Phänomen des Literarischen. Berlin/New York: de Gruyter 2009, S. 285-314. Boksers Streichquartett wird hingegen nicht als Leverkühns Komposition verkauft.

${ }^{119}$ Ebd., S. 3.

${ }^{120}$ Wolf: Narrativity in Instrumental Music?, S. 492.

121 „To Whom it May Concern“, ebd.

${ }^{122}$ Wolf: Narrativity in Instrumental Music?, ebd. 


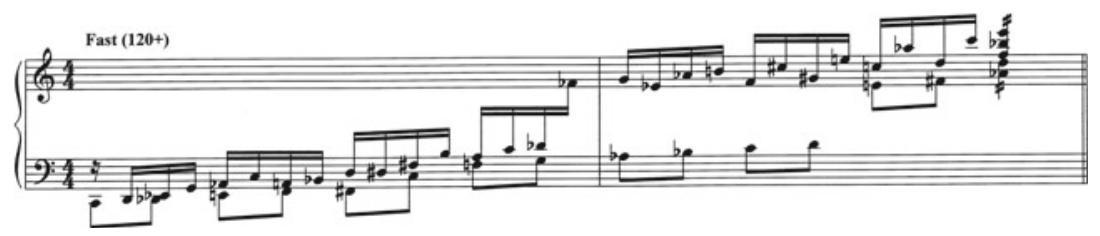

Abbildung 3.2 Odegards ,magical chords“ (S. 1 T. 1 f.)

ereignet sich alles in kurzer Zeit und es wird entsprechend schnell erzählt, was das rasch aufsteigende sexuelle Begehren zwischen den Geschwistern hervorhebt. Das musikalische Tempo passt sich der Schnelligkeit des Erzählten an. ${ }^{123}$

Nach den „magical chords“ kommt ein Duett zwischen Gregor und dem Fischer, der ihn ,sechzehn Meilen weit in die See hinaus zu einem flutumbrandeten Felsen“ (DF: 464) fährt, dort fesselt und den Schlüssel ins Meer wirft. Odegard erklärt, Leverkühns Text für diese Szene sei ,,clearly expected to shock the listener by portraying Gregory as an unwilling victim of forces behind his control“. 124 Nicht ohne Komik wird diese Szene realisiert:

Gregory: Oh dreary place!

Fisherman: What were you expecting?

G: A larger space?

F: There's room for genuflecting! (S. 1)

In Odegards Komposition scheint - wie der Text belegt - der Weg der Askese keine Konsequenz von Gregors freiem Willen zu sein. Hervorzuheben sind auch die Worte des Fischers, „no Seven-Eleven in heaven“ (S. 2 f.), die der Szene eine amerikanische Konnotation verleihen. Der Begriff „Seven-Eleven“, wie Odegard in den Anmerkungen, die dem Stück folgen, erläutert, bezieht sich auf eine Kette amerikanischer Convenience Stores, die von 7 bis 23 Uhr geöffnet sind und ,,convenience products“ at inflated prices“125 verkaufen. Der Fischer - so in den Selbsterläuterungen des Autors nach der Partitur - könnte hier auf die

${ }^{123}$ Bemerkenswert ist zudem, dass auch in Der Erwählte die Raschheit des sexuellen Aktes gleich nach dem Tod des Vaters zum Tragen kommt. Siehe Mann, T.: Der Erwählte, S. 30-36.

${ }^{124}$ „To Whom it May Concern“", S. 1.

${ }^{125}$ Ebd. 
späteren Ereignisse im Leben Gregors hindeuten und sich ihn als Papst vorstellen, der gegen Geld Sündenablässe verteilt. ${ }^{126}$ Auf das Duett folgt ein Rezitativ Gregors. Er wiederholt mehrfach „I repent!“ (S. 3); dann verspricht Gregor, „a good boy [...] for the rest of [...] [his] life" (S. 4) zu sein. Der Musikstil erinnert an den der italienischen Oper: In der Tat spielt Odegards The Calling hier auf die italienische Arie von Richard Strauss' Rosenkavalier an, die als ,musical ,intertextuality “"127 noch stärker die Verortung von Odegards Puppenspiel im Bereich der musikalischen Komödie verdeutlicht. ${ }^{128}$ Das Thema der Buße und Askese nimmt eine zentrale Stellung in Odegards Stück ein, wird aber durch die Lupe der Parodie interpretiert: Sowohl in den Selbsterläuterungen als auch im musikalischen Text wird eine gewisse Pauschalität und Naivität von religiösen Entscheidungen deutlich.

Der zweite Teil des Stückes (T. 1-53) schließt unmittelbar an den ersten an. Gregor ist verzweifelt, weil er denkt, dass er den Rest seines Lebens gefesselt verbringen wird und kein Ausweg aus der Verdammnis besteht: „Oh I am left here to die. [...] There is no hope left in me, there's no salvation!“ (S. 6 f.). Im Takt 21 fragt ihn aber ,,a sepulchral voice from on high“: „Gregory, do you repent now?“ (S. 7). Die Musik, die am Anfang ,sempre legato e appassionato con molto rubato“ (S. 6), also „immer legato und ausdrucksvoll mit viel Rubato“ zu spielen ist, wird im Takt 26 nach einer Passage mit Tremoli, die zum Spannungsaufbau beiträgt, und dem darauf folgenden Fortissimo zu einem „Slow Waltz“. Da beantwortet Gregor die Frage der Stimme von oben: „OH, LORD, gimme a break!“ (S. 7) (Abbildung 3.3).

In der Aufnahme bricht das Publikum in Gelächter aus. Zum einen wegen der Verwendung der Walzer-Form, gerade wenn Gregor mit Gott spricht, zum anderen, weil sich der aus edlem Blut geborene Papst umgangssprachlich äußert. Dies erinnert an Zeitbloms Beschreibung von Leverkühns Gesta, in denen ,das Skurrile [...] an die Stelle moralischer Priesterlichkeit trat" (DF: 466).

Odegard beschreibt den Stil der Komposition so: ${ }^{129}$

\footnotetext{
${ }^{126}$ Siehe ebd.

${ }^{127}$ Wolf: Narrativity in Instrumental Music?, S. 494.

${ }^{128}$ Siehe „To Whom it May Concern“, S. 1. und Strauss, Richard: Der Rosenkavalier. Komödie für Musik in drei Aufzügen von Hugo von Hofmannsthal, op. 59. Wien: Verlag Dr. Richard Strauss 1996, S. 113 f.

${ }^{129}$ Ebd.
} 


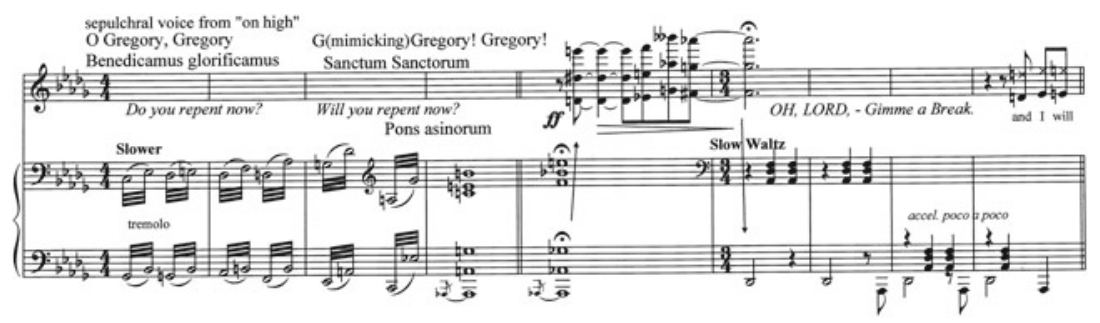

Abbildung 3.3 Gregors Gespräch mit Gott (S. 7 T. 21-29)

Little needs to be said about the style of the music. It is unabashedly romantic and certainly not yet dodecaphonic, although the chromatic complexity of the opening scene [...] has a motivic treatment that suggests some of the procedures of dodecaphonism.

Auch Leverkühn komponiert in dieser Phase noch nicht dodekaphonisch und sucht einen Weg zum Volk. Die Einbeziehung des Volks im musikalischen Vorhaben wird als das große Verdienst der Romantik betrachtet. ${ }^{130}$ The Calling of St. Gregory kann daher für eine intermediale Transposition gehalten werden, die sich stark an der Vorlage orientiert. Nichtsdestotrotz vertont Odegard nur einen Teil von Leverkühns Puppenspiel nach: Dementsprechend handelt es sich um eine partielle Vertonung des Romans selbst und eine partielle von Leverkühns Gesta. Zudem passt er den Stoff dem historisch-geographischen Kontext an, etwa durch den Sprachstil und den Bezug zu den convenience stores. Die Art der Intermedialität ist aufgrund der gleichzeitigen Präsenz der Medien Text und Musik als direct or overt einzustufen. Am Titel selbst lässt sich nicht gleich ablesen, dass das Werk auf Thomas Manns Roman Bezug nimmt: Er suggeriert zunächst einmal nur, dass er sich auf die spätmittelalterliche Exempelsammlung beziehen könnte. Die fehlende Erwähnung von Thomas Manns Doktor Faustus im Titel der Komposition schafft eine Kohärenz der paratextuellen Angaben, welche die Fiktion hervorrufen, dass Odegard Leverkühns Puppenspiel bearbeitet bzw. herausgegeben habe. Durch die Parodie interagiert The Calling simultan mit zwei textuellen Vorlagen, nämlich der Exempelsammlung und den Gesta-Kapiteln aus Doktor Faustus: Die spätmittelalterliche Vorlage wird wie im Roman durch Leverkühn in Frage gestellt und verschiedene Aspekte von Leverkühns Werk, speziell die

${ }^{130} \mathrm{Vgl}$. DF: $468 \mathrm{f}$. 
Parodie und die Dekonstruktion der Vorlage, werden in der Medienkombination verstärkt.

Es lohnt sich nun, kurz der Frage nachzugehen, wie das Inzest-Motiv bei Odegard interpretiert wird. In die Analyse können die bereits erwähnte Passage über die leidenschaftlichen und liebevollen Blicke von Gregors Eltern und eine weitere, in der Gregors Inzest mit seiner Mutter angesprochen wird sowie die folgenden Worte Gregors im Text: „I deserve to be alone, while I atone for my mother's erogenous zone“ (S. 2.) einbezogen werden. Das Inzest-Motiv unterliegt offenbar keiner „Selbstzensur des Textes“, ${ }^{131}$ es scheint kein Inzest-Tabu zu herrschen und das sexuelle Begehren kommt deutlich zum Ausdruck. ${ }^{132}$ Die Worte über die erogenen Zonen der Mutter machen deutlich, dass es sich um ein paritätisches Begehren und nicht um eines im Namen des übergeordneten Phallus handelt. ${ }^{133}$

Nicht nur der Inzest wird in The Calling entauratisiert, sondern auch, wie bereits angesprochen, die spätmittelalterliche Exempelsammlung mit ihrer moralisch-didaktischen Absicht, die durch das Vertonen von Leverkühns Werk zugleich „auf eine recht destruktive Weise“ (DF: 465) (teil-)reproduziert wird. „Were it not for Leverkuhn's [sic] well documented seriousness of purpose“, sagt Odegard „one might almost accuse him of writing purposeful kitsch to highlight the religious paradoxes that simpler faith does not question". ${ }^{134}$ Odegard situiert Leverkühns Werk an den Grenzen zum Kitsch; in seiner Komposition wird wie bei Leverkühn eine Gesellschaft für lächerlich erklärt, die blind an Mythen und pauschale religiöse Entscheidungen glaubt. Somit wird die gefährliche Seite des Mythos hervorgehoben, was in Doktor Faustus sowohl durch Leverkühns Vertonung der Gesta als auch beispielsweise durch den Kridwiß Kreis zum Tragen kommt. ${ }^{135}$ In den Sitzungen dieses Münchner Kreises, auf die das darauf folgende Kapitel eingeht, dient der Mythos zur Unterstützung politischer Stellungnahmen.

In Anlehnung an Braidotti erscheint in diesem Kapitel die Definition eines literarischen Textes als Labor, das Experimentieren voraussetzt und sichtbar macht, für die Analyse der Gesta-Kapitel von Thomas Manns Doktor Faustus besonders ergiebig. Einerseits eröffnet diese Auffassung neue Sichtweisen sowohl auf

\footnotetext{
${ }^{131}$ Frank: Inzest und Autor-Imago, S. 53.

${ }^{132}$ Auch in den Erläuterungen des Komponisten ist die Rede sowohl bezüglich des Geschwister- als auch des Eltern-Kind-Inzests von ,passionate and incestuous relationship“. Siehe „To Whom it May Concern“, S. 2.

${ }^{133}$ Siehe Butler: Antigone's Claim, S. 21.

${ }^{134}$ „To Whom it May Concern“, S. 3.

${ }^{135}$ Dies taucht allerdings im Gesamtwerk Thomas Manns auf. Siehe Schoene: „Ach, wäre fern, was ich liebe!“", S. 222.
} 
Adrian Leverkühns Puppenspiel Gesta romanorum als auch auf seinen Werdegang als Komponist, die weg von der Dichotomie Progression/Regression argumentieren und den Prozess besser in den Blick nehmen können. Andererseits treten Experimente und Transformationen zusätzlich auch aus werkexterner Sicht zutage, und zwar wenn man drei Werke Thomas Manns vergleicht, die sich mit Mythos und Inzest auseinandersetzen: die Novelle Wälsungenblut und die Romane Doktor Faustus und Der Erwählte. Auch die im zweiten Teil des Kapitels analysierten Kompositionen experimentieren mit ihrer Vorlage, den Gesta-Kapiteln von Doktor Faustus, und dementsprechend auch mit dem Mythos Gregor, dem Inzest und Leverkühns Ziel einer größeren Zugänglichkeit seiner musikalischen Sprache. Um diesem letzten Ziel nachzukommen, greift beispielsweise Beyer, der sonst zeit seines Lebens hauptsächlich absolute Musik komponiert, in Die Gesta romanorum - Musik des Adrian Leverkühn auf das Medium des Fernsehens zurück. Odegard konzentriert sich hingegen in The Calling of St. Gregory auf parodistische Aspekte der Vorlage, die selbst die moralische Absicht der spätmittelalterlichen Exempelsammlung umwertet, und „ent-auratisiert“ das Inzest-Motiv.

\subsection{Fazit}

Bereits diese erste Analyse zweier intermedialer Transpositionen nach Thomas Manns Roman, die von einer Untersuchung der transferierten Kapitel über Leverkühns Puppenspiel Gesta romanorum ausgehen, ließ durch die Auseinandersetzung mit der kompositorischen Rezeptionsgeschichte von Doktor Faustus einige neue Sichtweisen zu. Das Forschungsparadigma der Intermedialität half dabei, das Verhältnis der Kompositionen gegenüber der Vorlage auszuloten. Beyer verstärkt beispielsweise den Aspekt der Zugänglichkeit der Musik durch das Mediums des Fernsehens, indem er durch das angewandte Medium zugleich in Frage stellt, dass sich dieses Ziel Ende des 20. Jahrhunderts durch das Puppenspiel erreichen lässt. Odegard verstärkt die parodistischen Elemente der Vorlage, die er als nahezu kitschig interpretiert, so sehr, dass er eine Komposition schreibt, die auf Humor und Komik zurückgreift. Die Analyse sowohl der Effekte und Funktionen von Intermedialität als auch die Kontextualisierung des jeweiligen Werkes erwiesen sich als wichtige Werkzeuge des Medienvergleichs und speziell der Rekonstruktion der kompositorischen Rezeptionsgeschichte von Doktor Faustus. 
Die chronologische Behandlung von Leverkühns fiktiven Kompositionen setzt im nächsten Kapitel mit dem Oratorium Apocalipsis cum figuris fort. Zusammen mit der Dr. Fausti Weheklag wird diese Komposition durch die Erzählinstanz Zeitblom sehr ausführlich beschrieben und ermöglicht daher Leverkühns kompositorischen Werdegang noch genauer zu rekonstruieren. Einige in diesem Kapitel aufgetauchte Aspekte, z. B. Leverkühns Vorliebe für die Parodie und die Gefährlichkeit von Mythen, werden die Analyse auch im nächsten Kapitel begleiten und mit der Untersuchung des apokalyptischen Diskurses von Doktor Faustus und der Kompositionen kombiniert.

Open Access Dieses Kapitel wird unter der Creative Commons Namensnennung - Nicht kommerziell - Keine Bearbeitung 4.0 International Lizenz (http://creativecommons.org/lic enses/by-nc-nd/4.0/deed.de) veröffentlicht, welche die nicht-kommerzielle Nutzung, Vervielfältigung, Verbreitung und Wiedergabe in jeglichem Medium und Format erlaubt, sofern Sie den/die ursprünglichen Autor(en) und die Quelle ordnungsgemäß nennen, einen Link zur Creative Commons Lizenz beifügen und angeben, ob Änderungen vorgenommen wurden. Die Lizenz gibt Ihnen nicht das Recht, bearbeitete oder sonst wie umgestaltete Fassungen dieses Werkes zu verbreiten oder öffentlich wiederzugeben.

Die in diesem Kapitel enthaltenen Bilder und sonstiges Drittmaterial unterliegen ebenfalls der genannten Creative Commons Lizenz, sofern sich aus der Abbildungslegende nichts anderes ergibt. Sofern das betreffende Material nicht unter der genannten Creative Commons Lizenz steht und die betreffende Handlung nicht nach gesetzlichen Vorschriften erlaubt ist, ist auch für die oben aufgeführten nicht-kommerziellen Weiterverwendungen des Materials die Einwilligung des jeweiligen Rechteinhabers einzuholen.

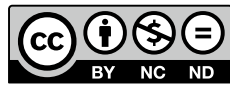

